\title{
Large-Scale, High-Resolution Comparison of the Core Visual Object Recognition Behavior of Humans, Monkeys, and State-of-the-Art Deep Artificial Neural Networks
}

\author{
-Rishi Rajalingham,* ๑Elias B. Issa,* Pouya Bashivan, Kohitij Kar, Kailyn Schmidt, and ๑James J. DiCarlo \\ McGovern Institute for Brain Research and Department of Brain and Cognitive Sciences, Massachusetts Institute of Technology, Cambridge, Massachusetts \\ 02139
}

Primates, including humans, can typically recognize objects in visual images at a glance despite naturally occurring identity-preserving image transformations (e.g., changes in viewpoint). A primary neuroscience goal is to uncover neuron-level mechanistic models that quantitatively explain this behavior by predicting primate performance for each and every image. Here, we applied this stringent behavioral prediction test to the leading mechanistic models of primate vision (specifically, deep, convolutional, artificial neural networks; ANNs) by directly comparing their behavioral signatures against those of humans and rhesus macaque monkeys. Using highthroughput data collection systems for human and monkey psychophysics, we collected more than one million behavioral trials from 1472 anonymous humans and five male macaque monkeys for 2400 images over 276 binary object discrimination tasks. Consistent with previous work, we observed that state-of-the-art deep, feedforward convolutional ANNs trained for visual categorization (termed DCNN $_{\text {IC }}$ models) accurately predicted primate patterns of object-level confusion. However, when we examined behavioral performance for individual images within each object discrimination task, we found that all tested $\mathrm{DCNN}_{\mathrm{IC}}$ models were significantly nonpredictive of primate performance and that this prediction failure was not accounted for by simple image attributes nor rescued by simple model modifications. These results show that current $\mathrm{DCNN}_{\mathrm{IC}}$ models cannot account for the image-level behavioral patterns of primates and that new ANN models are needed to more precisely capture the neural mechanisms underlying primate object vision. To this end, large-scale, high-resolution primate behavioral benchmarks such as those obtained here could serve as direct guides for discovering such models.

Key words: deep neural network; human; monkey; object recognition; vision

\section{Significance Statement}

Recently, specific feedforward deep convolutional artificial neural networks (ANNs) models have dramatically advanced our quantitative understanding of the neural mechanisms underlying primate core object recognition. In this work, we tested the limits of those ANNs by systematically comparing the behavioral responses of these models with the behavioral responses of humans and monkeys at the resolution of individual images. Using these high-resolution metrics, we found that all tested ANN models significantly diverged from primate behavior. Going forward, these high-resolution, large-scale primate behavioral benchmarks could serve as direct guides for discovering better ANN models of the primate visual system.

\section{Introduction}

Primates, both human and nonhuman, can typically recognize objects in visual images at a glance even in the face of naturally occurring identity-preserving transformations such as changes in viewpoint. This view-invariant visual object recognition ability is thought to be supported primarily by the primate ventral visual stream (Tanaka, 1996; Rolls, 2000; DiCarlo et al., 2012). A pri-

E. Issa's present address: Department of Neuroscience, Zuckerman Mind Brain Behavior Institute, Columbi University, New York, NY 10027.

Correspondence should be addressed to James J. DiCarlo, McGovern Institute for Brain Research, Department of Brain and Cognitive Sciences, Massachusetts Institute of Technology, MIT 46-6161, 43 Vassar Street, Cambridge, MA 02139. E-mail: dicarlo@mit.edu.

DOI:10.1523/JNEUROSCI.0388-18.2018

Copyright $\odot 2018$ the authors $\quad 0270-6474 / 18 / 387255-15 \$ 15.00 / 0$ 
mary neuroscience goal is to construct computational models that quantitatively explain the neural mechanisms underlying this ability. That is, our goal is to discover artificial neural networks (ANNs) that accurately predict neuronal firing rate responses at all levels of the ventral stream and its behavioral output. To this end, specific models within a large family of deep, convolutional neural networks (DCNNs), optimized by supervised training on large-scale category-labeled image sets (ImageNet) to match human-level categorization performance (Krizhevsky et al., 2012; LeCun et al., 2015), have been put forth as the leading ANN models of the ventral stream (Kriegeskorte, 2015; Yamins and DiCarlo, 2016). We refer to this subfamily as DCNN $_{\text {IC }}$ models (IC to denote ImageNet categorization pretraining) to distinguish them from all possible models in the DCNN family and, more broadly, from the superfamily of all ANNs. To date, it has been shown that $\mathrm{DCNN}_{\mathrm{IC}}$ models display internal feature representations similar to neuronal representations along the primate ventral visual stream (Yamins et al., 2013, 2014; Cadieu et al., 2014; Khaligh-Razavi and Kriegeskorte, 2014) and they exhibit behavioral patterns similar to the behavioral patterns of pairwise object confusions of primates (Ghodrati et al., 2014; Rajalingham et al., 2015; Jozwik et al., 2016; Kheradpisheh et al., 2016). Therefore, $\mathrm{DCNN}_{\mathrm{IC}}$ models may provide a quantitative account of the neural mechanisms underlying primate core object recognition behavior.

However, several studies have shown that $\mathrm{DCNN}_{\mathrm{IC}}$ models can diverge drastically from humans in object recognition behavior, especially with regard to particular images optimized to be adversarial to these networks (Goodfellow et al., 2014; Nguyen et al., 2015). Related work has shown that specific image distortions are disproportionately challenging to current DCNNs compared with humans (RichardWebster et al., 2016; Dodge and Karam, 2017; Geirhos et al., 2017; Hosseini et al., 2017). Such imagespecific failures of the current ANN models would likely not be captured by "object-level” behavioral metrics (e.g., the pattern of pairwise object confusions mentioned above) that are computed by pooling over hundreds of images and thus are not sensitive to variation in difficulty across images of the same object. To overcome this limitation of prior work, we here aimed to use scalable behavioral testing methods to precisely characterize primate behavior at the resolution of individual images and to directly compare leading DCNN models to primates over the domain of core object recognition behavior at this high resolution.

We focused on core invariant object recognition, the ability to identify objects in visual images in the central visual field during a single, natural viewing fixation (DiCarlo et al., 2012). We further restricted our behavioral domain to basic-level object discriminations, as defined previously (Rosch et al., 1976). Within this domain, we collected large-scale, high-resolution measurements of human and monkey behavior (over a million behavioral trials) using high-throughput psychophysical techniques, including a novel home cage behavioral system for monkeys. These data enabled us to systematically compare all systems at progressively higher resolution. At lower resolutions, we replicated previous findings that humans, monkeys, and $\mathrm{DCNN}_{\mathrm{IC}}$ models all share a common pattern of object-level confusion (Rajalingham et al., 2015). However, at the higher resolution of individual images, we found that the behavior of all tested $\mathrm{DCNN}_{\mathrm{IC}}$ models was significantly different from human and monkey behavior and this model prediction failure could not be easily rescued by simple model modifications. These results show that current $\mathrm{DCNN}_{\mathrm{IC}}$ models do not fully account for the image-level behavioral patterns of primates, suggesting that new ANN models are needed to more precisely capture the neural mechanisms underlying primate object vision. To this end, large-scale high-resolution behavioral benchmarks such as those obtained here could serve as a strong top-down constraint for efficiently discovering such models.

\section{Materials and Methods}

Visual images. We examined basic-level, core object recognition behavior using a set of 24 broadly sampled objects that we previously found to be reliably labeled by independent human subjects based on the definition of basic level proposed previously (Rosch et al., 1976). For each object, we generated 100 naturalistic synthetic images by first rendering a 3D model of the object with randomly chosen viewing parameters (2D position, $3 \mathrm{D}$ rotation, and viewing distance) and then placing that foreground object view onto a randomly chosen, natural image background. To do this, each object was first assigned a canonical position (center of gaze), scale $\left(\sim 2^{\circ}\right)$, and pose and then its viewing parameters were randomly sampled uniformly from the following ranges for object translation $\left([-3,3]^{\circ}\right.$ in both $\mathrm{h}$ and $\mathrm{v})$, rotation $\left([-180,180]^{\circ}\right.$ in all three axes $)$, and scale $([\times 0.7$, $\times 1.7])$. Background images were sampled randomly from a large database of high-dynamic range images of indoor and outdoor scenes obtained from Dosch Design (www.doschdesign.com). This image generation procedure enforces invariant object recognition rather than image matching because it requires the visual recognition system (human, animal or model) to tackle the "invariance problem," the computational crux of object recognition (Ullman and Humphreys, 1996; Pinto et al., 2008). In particular, we used naturalistic synthetic images with systematic variation in viewpoint parameters and uncorrelated background to remove potential confounds (natural images are often "composed" such that backgrounds covary with the object category) while keeping the task difficult for machine vision systems. We have previously shown that, unlike some photographic image sets, synthetic images of the types we used here are critical to separating some types of computer vision systems from humans (Pinto et al., 2008). Using this procedure, we previously generated 2400 images ( 100 images per object) rendered at $1024 \times 1024$ pixel resolution with 256 -level gray scale and subsequently resized to $256 \times 256$ pixel resolution for human psychophysics, monkey psychophysics, and model evaluation (Rajalingham et al., 2015). In the current work, we focused our analyses on a randomly subsampled, and then fixed, subset of 240 images ( 10 images per object; here referred to as the "primary test images"). Figure $1 A$ shows the full list of 24 objects, with two example images of each object.

Because all of the images were generated from synthetic 3D object models, we had explicit knowledge of the viewpoint parameters (position, size, and pose) for each object in each image, as well as perfect segmentation masks. Taking advantage of this feature, we characterized each image based on these high-level attributes consisting of size, eccentricity, relative pose, and contrast of the object in the image. Note that these meta-parameters were independently randomly sampled to generate each image, so there is no correlation among size, eccentricity, and pose over images. The size and eccentricity of the object in each image were computed directly from the corresponding viewpoint parameters under the assumption that the entire image would subtend $6^{\circ}$ at the center of visual gaze ( $\pm 3^{\circ}$ in both azimuth and elevation; see below). For each synthetic object, we first defined its "canonical" 3D pose vector based on independent human judgments. To compute the relative pose attribute of each image, we estimated the difference between the object's $3 \mathrm{D}$ pose and its canonical 3D pose. Pose differences were computed as distances in unit quaternion representations: the $3 \mathrm{D}$ pose $\left(r_{\mathrm{xy}}, r_{\mathrm{xz}}\right.$, and $r_{\mathrm{yz}}$ ) was first converted into unit quaternions and distances between quaternions $q_{1}$ and $q_{2}$ were estimated as $\cos ^{-1}\left|q_{1} \cdot q_{2}\right|$ (Huynh, 2009). To compute the object contrast, we measured the absolute difference between the mean of the pixel intensities corresponding to the object and the mean of the background pixel intensities in the vicinity of the object (specifically, within 25 pixels of any object pixel, analogous to computing the local foreground-background luminance difference of a foreground object in an image). Figure $5 \mathrm{~A}$ shows example images with varying values for the four image attributes. 

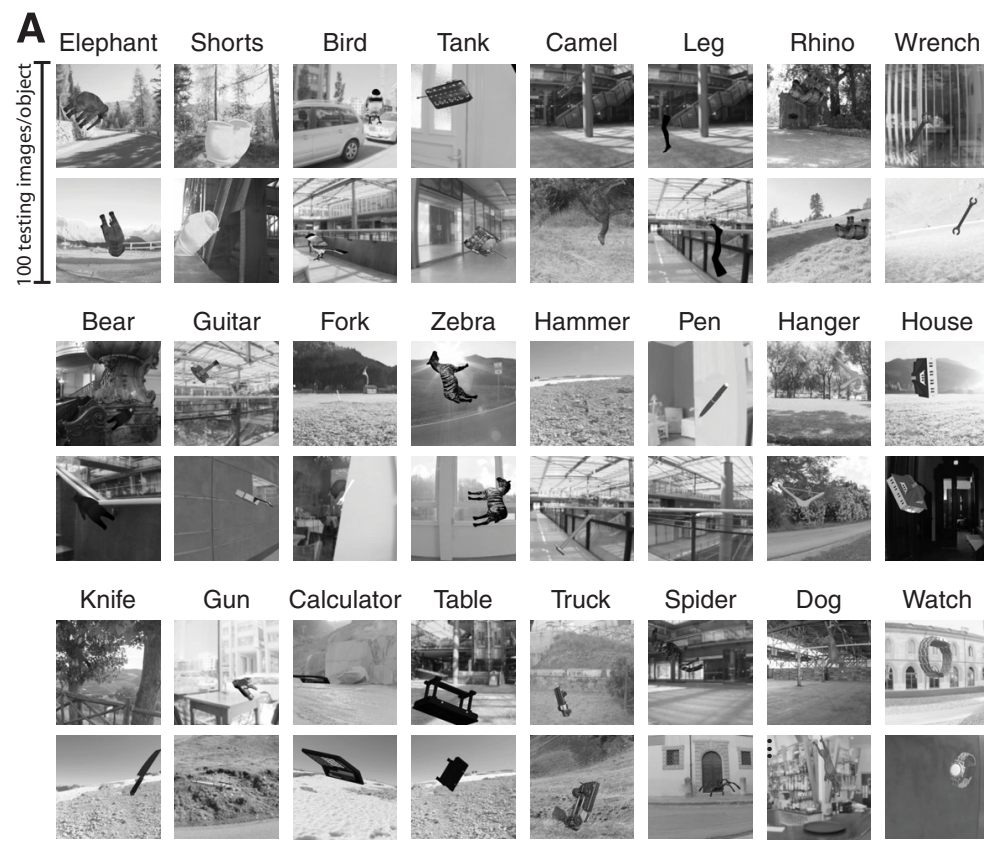

Watch

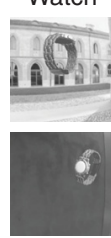

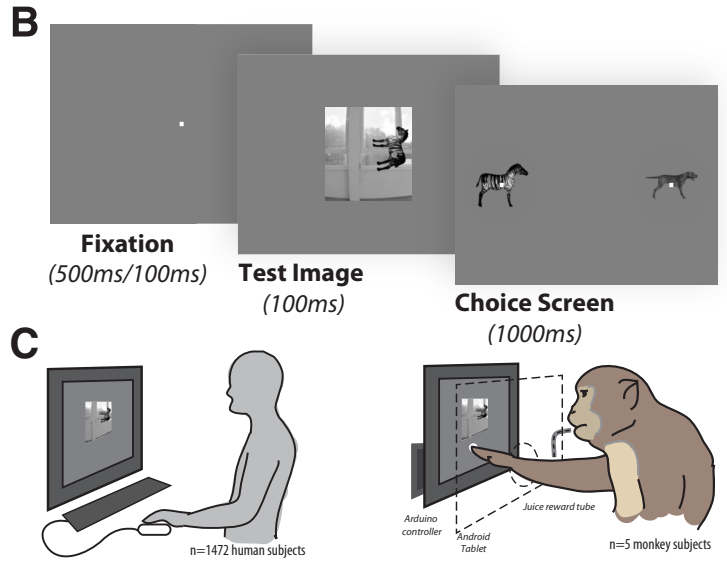

Human Behavior (Mechanical Turk)

Monkey Behavior (Monkey-Turk)

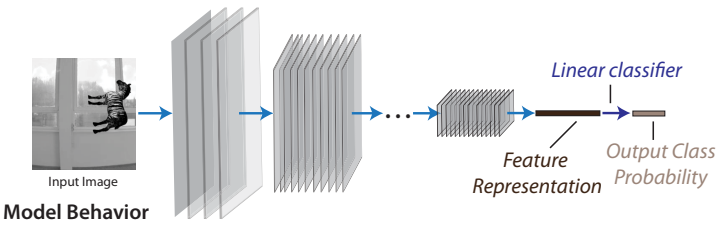

Figure 1. Images and behavioral task. $\boldsymbol{A}$, Two (of 100) example images for each of the 24 basic-level objects. To enforce true invariant object recognition behavior, we generated naturalistic synthetic images, each with one foreground object, by rendering a 3D model of each object with randomly chosen viewing parameters and placing that foreground object view onto a randomly chosen, natural image background. $\boldsymbol{B}$, Time course of example behavioral trial (zebra vs dog) for human psychophysics. Each trial initiated with a central fixation point for 500 ms, followed by 100 ms presentation of a square test image (spanning $6-8^{\circ}$ of visual angle). After extinction of the test image, two choice images were shown to the left and right. Human participants were allowed to freely view the response images for up to $1000 \mathrm{~ms}$ and responded by clicking on one of the choice images; no feedback was given. To neutralize top-down feature attention, all 276 binary object discrimination tasks were randomly interleaved on a trial-by-trial basis. The monkey task paradigm was nearly identical to the human paradigm with the exception that trials were initiated by touching a fixation circle horizontally centered on the bottom third of the screen and successful trials were rewarded with juice, whereas incorrect choices resulted in timeouts of $1-2.5 \mathrm{~s}$. $\boldsymbol{C}$, Large-scale and high-throughput psychophysics in humans (top left), monkeys (top right), and models (bottom). Human behavior was measured using the online Amazon MTurk platform, which enabled the rapid collection of $\sim 1$ million behavioral trials from 1472 human subjects. Monkey behavior was measured using a novel custom home cage behavioral system (MonkeyTurk), which leveraged a web-based behavioral task running on a tablet to test many monkey subjects simultaneously in their home environment. Deep convolutional neural network models were tested on the same images and tasks as those presented to humans and monkeys by extracting features from the penultimate layer of each visual system model and training back-end multiclass logistic regression classifiers. All behavioral predictions of each visual system model were for images that were not seen in any phase of model training.

Core object recognition behavioral paradigm. Core object discrimination is defined as the ability to discriminate between two or more objects in visual images presented under high view uncertainty in the central visual field $\left(\sim 10^{\circ}\right)$ for durations that approximate the typical primate free-viewing fixation duration ( $200 \mathrm{~ms}$ ) (DiCarlo and Cox, 2007; DiCarlo et al., 2012). As in our previous work (Rajalingham et al., 2015), the behavioral task paradigm consisted of a interleaved set of binary discrimination tasks. Each binary discrimination task is an object discrimination task between a pair of objects (e.g., elephant vs bear). Each such binary task is balanced in that the test image is equally likely (50\%) to be of either of the two objects. On each trial, a test image is presented, followed by a choice screen showing canonical views of the two possible objects (the object that was not displayed in the test image is referred to as the "distractor" object, but note that objects are equally likely to be distractors and targets). Here, 24 objects were tested, which resulted in 276 binary object discrimination tasks. To neutralize feature attention, these 276 tasks are randomly interleaved (trial by trial) and the global task is referred to as a basic-level, core object recognition task paradigm.

Testing human behavior. All human behavioral data presented here were collected from 1476 anonymous human subjects on Amazon Mechanical Turk (MTurk) performing the task paradigm described above. Subjects were instructed to report the identity of the foreground object in each presented image from among the two objects presented on the choice screen (see Fig. 1B). Because all 276 tasks were interleaved randomly (trial by trial), subjects could not deploy feature attentional strategies specific to each object or specific to each binary task to process each test image.

Figure $1 B$ illustrates the time course of each behavioral trial, for a particular object discrimination task (e.g., zebra vs dog). Each trial initiated with a central black point for $500 \mathrm{~ms}$, followed by $100 \mathrm{~ms}$ presenta- tion of a test image containing one foreground object presented under high variation in viewing parameters and overlaid on a random background, as described above (see "Visual images" section above). Immediately after extinction of the test image, two choice images, each displaying a single object in a canonical view with no background, were shown to the left and right. One of these two objects was always the same as the object that generated the test image (i.e., the correct object choice) and the location of the correct object (left or right) was randomly chosen on each trial. After clicking on one of the choice images, the subject was queued with another fixation point before the next test image appeared. No feedback was given and human subjects were never explicitly trained on the tasks. Under assumptions of typical computer ergonomics, we estimate that images were presented at a $6-8^{\circ}$ of visual angle at the center of gaze and the choice object images were presented at $\pm 6-8^{\circ}$ of eccentricity along the horizontal meridian.

We measured human behavior using the online Amazon MTurk platform (see Fig. 1C), which enables efficient collection of large-scale psychophysical data from crowd-sourced "human intelligence tasks" (HITs). The reliability of the online MTurk platform has been validated by comparing results obtained from online and in-laboratory psychophysical experiments (Majaj et al., 2015; Rajalingham et al., 2015). We pooled 927,296 trials from 1472 human subjects to characterize the aggregate human behavior, which we refer to as the "pooled" human (or "archetypal" human). Each human subject performed only a small number of trials $(\sim 150)$ on a subset of the images and binary tasks. All 2400 images were used for behavioral testing but, in some of the HITs, we biased the image selection toward the 240 primary test images ( $1424 \pm 70$ trials/image on this subsampled set vs $271 \pm 93$ trials/image on the remaining images, mean \pm SD) to efficiently characterize behavior at image-level resolution. Images were randomly drawn such that each hu- 
man subject was exposed to each image a relatively small number of times $(1.5 \pm 2.0$ trials/image per subject, mean $\pm \mathrm{SD})$, to mitigate potential alternative behavioral strategies (e.g., "memorization" of images) that could arise from a finite image set. Behavioral signatures at the objectlevel (B.O1, B.O2, see "Behavioral metrics and signatures" section) were measured using all 2400 test images, whereas image-level behavioral signatures (B.I1n, B.I2n, see "Behavioral metrics and signatures" section) were measured using the 240 primary test images. (We observed qualitatively similar results using those metrics on the full 2400 test images, but we here focus on the primary test images because the larger number of trials leads to lower noise levels).

Five other human subjects were separately recruited on MTurk to each perform a large number of trials on the same images and tasks $(53,097 \pm$ 15,278 trials/subject, mean \pm SD). Behavioral data from these five subjects was not included in the characterization of the pooled human described above, but instead aggregated together to characterize a distinct held-out human pool. For the scope of the current work, this held-out human pool, which largely replicated all behavioral signatures of the larger archetypal human (see Figs. 2 and 3), served as an independent validation of our human behavioral measurements.

Testing monkey behavior. Five adult male rhesus macaque monkeys (Macaca mulatta, subjects M, Z, N, P, and B) were tested on the same basiclevel, core object recognition task paradigm described above, with minor modification as described below. All procedures were performed in compliance with National Institutes of Health guidelines and the standards of the Massachusetts Institute of Technology Committee on Animal Care and the American Physiological Society. To efficiently characterize monkey behavior, we used a novel home cage behavioral system that we developed (termed MonkeyTurk; see Fig. 1C). This system leveraged a tablet touchscreen (9inch Google Nexus or 10.5-inch Samsung Galaxy Tab S) and used a web application to wirelessly load the task and collect the data (code available from https://github.com/dicarlolab/mkturk). Analogous to the online MTurk, which allows for efficient psychophysical assays of a large number (hundreds) of human users in their native environments, MonkeyTurk allowed us to test many monkey subjects simultaneously in their home environment. Each monkey voluntarily initiated trials and each readily performed the task a few hours each day that the task apparatus was made available to it. At an average rate of $\sim 2000$ trials per day per monkey, we collected a total of 836,117 trials from the 5 monkey subjects over a period of $\sim 3$ months.

Monkey training was described in detail previously (Rajalingham et al., 2015). Briefly, all monkeys were initially trained on the match test image to object rule using other images and were also trained on discriminating the particular set of 24 objects tested here using a separate set of training images rendered from these objects in the same manner as the main testing images. Two of the monkey subjects $(\mathrm{Z}$ and $\mathrm{M})$ were previously trained in the laboratory setting and the remaining three subjects were trained using MonkeyTurk directly in their home cages and did not have significant prior laboratory exposure. Once monkeys reached saturation performance on training images, we began the behavioral testing phase to collect behavior on test images. Monkeys did improve throughout the testing phase, exhibiting an increase in performance between the first and second half of trials of $4 \pm 0.9 \%$ (mean \pm SEM over five monkey subjects). However, the image-level behavioral signatures obtained from the first and the second halves of trials were highly correlated to each other (B.I1 noise-adjusted correlation of $0.85 \pm 0.06$, mean \pm SEM over five monkey subjects, see "Behavioral metrics and signatures" section below), suggesting that monkeys did not significantly alter strategies (e.g., did not "memorize" images) throughout the behavioral testing phase.

The monkey task paradigm was nearly identical to the human paradigm (see Fig. $1 B$ ) with the exception that trials were initiated by touching a white "fixation" circle horizontally centered on the bottom third of the screen (to avoid occluding centrally presented test images with the hand). This triggered a $100 \mathrm{~ms}$ central presentation of a test image, followed immediately by the presentation of the two choice images (see Fig. $1 B$, location of correct choice randomly assigned on each trial, identical to the human task). Unlike the main human task, monkeys responded by directly touching the screen at the location of one of the two choice images. Touching the choice image corresponding to the object shown in the test image resulted in the delivery of a drop of juice through a tube positioned at mouth height (but not obstructing view), whereas touching the distractor choice image resulted in a $3 \mathrm{~s} \mathrm{timeout.} \mathrm{Because} \mathrm{gaze} \mathrm{direc-}$ tion typically follows the hand during reaching movements, we assumed that the monkeys were looking at the screen during touch interactions with the fixation or choice targets. In both the laboratory and in the home cage, we maintained total test image size at $\sim 6^{\circ}$ of visual angle at the center of gaze and took advantage of the retina-like display qualities of the tablet by presenting images pixel matched to the display $(256 \times 256$ pixel image displayed using $256 \times 256$ pixels on the tablet at a distance of 8 inches) to avoid filtering or aliasing effects.

As with Mechanical Turk testing in humans, MonkeyTurk head-free home cage testing enables efficient collection of reliable, large-scale psychophysical data, but it likely does not yet achieve the level of experimental control that is possible in the head-fixed laboratory setting. However, we note that when subjects were engaged in home cage testing, they reliably had their mouth on the juice tube and their arm positioned through an armhole. These spatial constraints led to a high level of head position trial-by-trial reproducibility during performance of the task paradigm. Furthermore, when subjects were in this position, they could not see other animals because the behavior box was opaque and subjects performed the task at a rapid pace of 40 trials/min, suggesting that they were not frequently distracted or interrupted. The location of the upcoming test image (but not the location of the object within that test image) was perfectly predictable at the start of each behavioral trial, which likely resulted in a reliable, reproduced gaze direction at the moment that each test image was presented. The relatively short, but natural and high performing (Cadieu et al., 2014), test image duration (100 ms) ensured that saccadic eye movements were unlikely to influence test image performance because they generally take $\sim 200 \mathrm{~ms}$ to initiate in response to the test image and thus well after the test image had been extinguished.

Testing model behavior. We tested a number of different DCNN models on the exact same images and tasks as those presented to humans and monkeys. Importantly, our core object recognition task paradigm is closely analogous to the large-scale ImageNet 1000-way object categorization task for which these networks were optimized and thus expected to perform well. We focused on publicly available DCNN model architectures that have proven highly successful with respect to this computer vision benchmark over the past 5 years: AlexNet (Krizhevsky et al., 2012), NYU (Zeiler and Fergus, 2014), VGG (Simonyan and Zisserman, 2014), GoogleNet (Szegedy et al., 2013), Resnet (He et al., 2016), and Inception-v3 (Szegedy et al., 2013). Because this is only a subset of possible DCNN models, we refer to these as the $\mathrm{DCNN}_{\mathrm{IC}}$ visual system model subfamily. For each of the publicly available model architectures, we first used ImageNet categorization-trained model instances either using publicly available trained model instances or training them to saturation on the 1000-way classification task in-house. Training took several days on one to two GPUs.

We then performed psychophysical experiments on each ImageNettrained DCNN model to characterize their behavior on the exact same images and tasks as humans and monkeys. We first adapted these ImageNet-trained models to our 24-way object recognition task by retraining the final class probability layer (initially corresponding to the probability output of the 1000-way ImageNet classification task) while holding all other layers fixed. In practice, this was done by extracting features from the penultimate layer of each $\mathrm{DCNN}_{\mathrm{IC}}$ (i.e., top-most before class probability layer) on the same images that were presented to humans and monkeys and training back-end multiclass logistic regression classifiers to determine the cross-validated output class probability for each image. This procedure is illustrated in Figure 1C. To estimate the hit rate of a given image in a given binary classification task, we renormalized the 24-way class probabilities of that image, considering only the two relevant classes, to sum to one. Object-level and image-level behavioral metrics were computed based on these hit rate estimates (as described in the "Behavioral metrics and signatures" section below). Importantly, this procedure assumes that the model "retina" layer processes the central $6^{\circ}$ of the visual field. It also assumes that linear discrim- 
Table 1. Definition of behavioral performance metrics

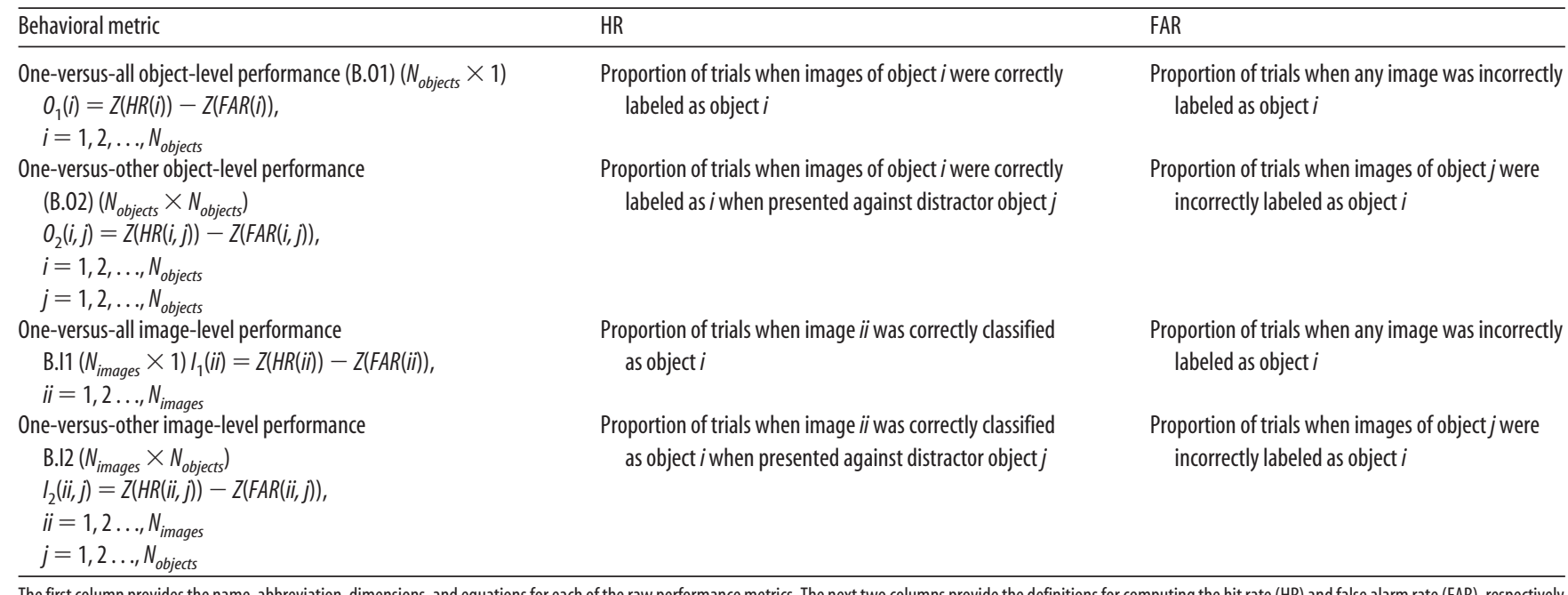

The first column provides the name, abbreviation, dimensions, and equations for each of the raw performance metrics. The next two columns provide the definitions for computing the hit rate (HR) and false alarm rate (FAR), respectively.

inants ("readouts") of the model's top feature layer are its behavioral output (as intended by the model designers). Manipulating either of these choices (e.g., resizing the input images such that they span only part of the input layer or building linear discriminates for behavior using a different model feature layer) would result in completely new, testable ANN models that we did not test here.

From these analyses, we selected the most human consistent $\mathrm{DCNN}_{\mathrm{IC}}$ architecture (Inception-v3, see "Behavioral consistency" section below), fixed that architecture, and then performed post hoc analyses in which we varied the input image sampling, the initial parameter settings before training, the filter training images, the type of classifiers used to generate the behavior from the model features, and the classifier training images. To examine input image sampling, we retrained the Inception-v3 architecture on images from ImageNet that were first spatially filtered to match the spatial sampling of the primate retina (i.e., an approximately exponential decrease in cone density away from the fovea) by effectively simulating a fisheye transformation on each image. These images were at highest resolution at the "fovea" (i.e., center of the image) with gradual decrease in resolution with increasing eccentricity. To examine the analog of "intersubject variability," we constructed multiple trained model instances ("subjects") in which the architecture and training images were held fixed (Inception-v3 and ImageNet, respectively) but the model filter weights initial condition and order of training images were randomly varied for each model instance. Importantly, this procedure is only one possible choice for simulating intersubject variability for DCNN models, which, as a first-order approximation, models different human subjects as random samples from a fixed model class. There are many other possible options for simulating intersubject variability, including: (1) random sampling of different features from a fixed trained model of fixed architecture; (2) random sampling of different trained models from a fixed architecture and optimization; and (3) random sampling of different trained models from a model class varying in architecture (3a), optimization procedure and data $(3 \mathrm{~b})$, or both $(3 \mathrm{c})$, to name a few. This choice is an important open research direction that we do not address here. We do not claim this to be the best model of intersubject variability, but rather a good starting point for that greater goal.

To examine the effect of model training, we first fine-tuned an ImageNet-trained Inception-v3 model on a synthetic image set consisting of $\sim 6.9$ million images of 1049 objects (holding out 50,000 images for model validation). These images were generated using the same rendering pipeline as our test images, but the objects were nonoverlapping with the 24 test objects presented here. As expected, fine-tuning on synthetic images led to a small increase in overall performance (see Fig. 5). To push the limits of training on synthetic images, we additionally trained an Inception-v3 architecture exclusively on this synthetic image set. To avoid overfitting on this synthetic image set, we stopped the model training based on maximum performance on a validation set of held-out objects. These synthetic-trained models were high performing, with only a small decrease in overall performance relative to the ImageNet-trained models (see Fig. 5, last bar). Finally, we tested the effect of different classifiers to generate model behavior by testing both multiclass logistic regression and support vector machine classifiers as well as the effect of varying the number of training images used to train those classifiers (20 vs 50 images per class).

Behavioral metrics and signatures. To characterize the behavior of any visual system, we here introduce four behavioral $(B)$ metrics of increasing richness requiring increasing amounts of data to measure reliably. Each behavioral metric computes a pattern of unbiased behavioral performance, using a sensitivity index: $d^{\prime}=Z$ (HitRate) $-Z$ (FalseAlarmRate), where $Z$ is the inverse of the cumulative Gaussian distribution. The various metrics differ in the resolution at which hit rates and false alarm rates are computed. Table 1 summarizes the four behavioral metrics, varying the hit rate resolution (object-level or image-level) and the false alarm resolution (one-versus-all or one-versus-other). When each metric is applied to the behavioral data of a visual system, biological or artificial, we refer to the result as one behavioral "signature" of that system. Note that we do not consider the signatures obtained here to be the final say on the behavior of these biological or artificial systems, in the terms defined here, new experiments using new objects/images, but the same metrics would produce additional behavioral signatures.

The four behavioral metrics we chose are as follows. First, the oneversus-all object-level performance metric (termed B.O1) estimates the discriminability of each object from all other objects, pooling across all distractor object choices. Because we here tested 24 objects, the resulting B.O1 signature has 24 independent values. Second, the one-versus-other object-level performance metric (termed B.O2) estimates the discriminability of each specific pair of objects or the pattern of pairwise object confusions. Because we here tested 276 interleaved binary object discrimination tasks, the resulting B.O2 signature has 276 independent values (the off-diagonal elements on one-half of the $24 \times 24$ symmetric matrix). Third, the one-versus-all image-level performance metric (termed B.I1) estimates the discriminability of each image from all other objects, pooling across all possible distractor choices. Because we here focused on the primary image test set of 240 images ( 10 per object, see above), the resulting B.I1 signature has 240 independent values. Fourth, the oneversus-other image-level performance metric (termed B.I2) estimates the discriminability of each image from each distractor object. Because we here focused on the primary image test set of 240 images ( 10 per object, see above) with 23 distractors, the resulting B.I2 signature has 5520 independent values.

Naturally, object-level and image-level behavioral signatures are tightly linked. For example, images of a particularly difficult-to-discriminate object would inherit lower performance values on average compared with images from a less difficult-to-discriminate object. To isolate the 
behavioral variance that is specifically driven by image variation and not simply predicted by the objects (and thus already captured by B.O1 and B.O2), we defined normalized image-level behavioral metrics (termed B.I1n, B.I2n) by subtracting the mean performance values over all images of the same object and task. This process is schematically illustrated in Figure $3 \mathrm{~A}$. We note that the resulting normalized image-level behavioral signatures capture a significant proportion of the total image-level behavioral variance in our data (e.g., 52\%, 58\% of human B.I1 and B.I2 variance is driven by image variation, independent of object identity). In this study, we use these normalized metrics for image-level behavioral comparisons between models and primates (see Results).

Behavioral consistency. To quantify the similarity between a model visual system and the human visual system with respect to a given behavioral metric, we used a measure called "human consistency" as previously defined (Johnson et al., 2002). Human consistency ( $\tilde{\boldsymbol{\rho}})$ is computed, for each of the four behavioral metrics, as a noise-adjusted correlation of behavioral signatures (DiCarlo and Johnson, 1999). For each visual system, we randomly split all behavioral trials into two equal halves and applied each behavioral metric to each half, resulting in two independent estimates of the system's behavioral signature with respect to that metric. We took the Pearson correlation between these two estimates of the behavioral signature as a measure of the reliability of that behavioral signature given the amount of data collected; that is, the split-half internal reliability. To estimate human consistency, we computed the Pearson correlation over all the independent estimates of the behavioral signature from the model $(\boldsymbol{m})$ and the human $(\boldsymbol{h})$ and we then divide that raw Pearson correlation by the geometric mean of the split-half internal reliability of the same behavioral signature measured for each system as follows: $\tilde{\rho}(m, h)=\frac{\rho(\boldsymbol{m}, \boldsymbol{h})}{\sqrt{\rho(\boldsymbol{m}, \boldsymbol{m}) \rho(\boldsymbol{h}, \boldsymbol{h})}}$.

Because all correlations in the numerator and denominator were computed using the same amount of trial data (exactly half of the trial data), we did not need to make use of any prediction formulas (e.g., extrapolation to larger number of trials using Spearman-Brown prediction formula). This procedure was repeated 10 times with different random split-halves of trials. Our rationale for using a reliability-adjusted correlation measure for human consistency was to account for variance in the behavioral signatures that arises from "noise"; that is, variability that is not replicable by the experimental condition (image and task) and thus that no model can be expected to predict (DiCarlo and Johnson, 1999; Johnson et al., 2002). In sum, if the model $(m)$ is a replica of the archetypal human $(h)$, then its expected human consistency is 1.0 regardless of the finite amount of data that are collected. Note that the human consistency value is directly linked, via a squaring operation, to the proportion of the explainable behavioral variance that is explained by models.

Characterization of residuals. In addition to measuring the similarity between the behavioral signatures of primates and models (using human consistency analyses, as described above), we examined the corresponding differences, termed "residual signatures." Each candidate visual system model's residual signature was estimated as the residual of a linear least-squares regression of the model's signature on the corresponding human signature and a free intercept parameter. This procedure effectively captures the differences between human and model signatures after accounting for overall performance differences. Residual signatures were estimated on disjoint split-halves of trials repeated 10 times with random trial permutations. Residuals were computed with respect to the normalized one-versus-all image-level performance metric (B.I1n) because this metric showed a clear difference between $\mathrm{DCNN}_{\mathrm{IC}}$ models and primates and the behavioral residual can be interpreted based only on the test images (i.e., we can assign a residual per image).

To examine the extent to which the difference between each model and the archetypal human is reliably shared across different models, we measured the Pearson correlation between the residual signatures of pairs of models. Residual similarity was quantified as the proportion of shared variance, defined as the square of the noise-adjusted correlation between residual signatures (the noise adjustment was done as defined in equation above). Correlations of residual signatures were always computed across distinct split-halves of data to avoid introducing spurious correlations from subtracting common noise in the human data. We mea- sured the residual similarity between all pairs of tested models, holding both architecture and optimization procedure fixed (between instances of the ImageNet categorization-trained Inception-v3 model varying in filter initial conditions), varying the architecture while holding the optimization procedure fixed (between all tested ImageNet categorizationtrained DCNN architectures), and holding the architecture fixed while varying the optimization procedure (between ImageNet categorizationtrained Inception-v3 and synthetic categorization fine-tuned Inception-v3 models). This analysis addresses not only the reliability of the failure of $\mathrm{DCNN}_{\mathrm{IC}}$ models to predict human behavior (deviations from humans), but also the relative importance of the characteristics defining similarities within the model subfamily (namely, the architecture and the optimization procedure). We first performed this analysis for residual signatures over the 240 primary test images and subsequently zoomed in on subsets of images that humans found to be particularly difficult. This image selection was made relative to the distribution of image-level performance of held-out human subjects (B.I1 metric from five subjects); difficult images were defined as ones with performance below the $25^{\text {th }}$ percentile of this distribution.

To determine whether the difference between each model and humans can be explained by simple human-interpretable stimulus attributes, we regressed each $\mathrm{DCNN}_{\mathrm{IC}}$ model's residual signature on image attributes (object size, eccentricity, pose, and contrast). Briefly, we constructed a design matrix from the image attributes (using individual attributes or all attributes) and used multiple linear least-squares regression to predict the image-level residual signature. The multiple linear regression was tested using twofold cross-validation over trials. The relative importance of each attribute (or groups of attributes) was quantified using the proportion of explainable variance (i.e., variance remaining after accounting for noise variance) explained from the residual signature.

Primate behavior zone. In this work, we are primarily concerned with the behavior of an "archetypal human" rather than the behavior of any given individual human subject. We operationally defined this concept as the common behavior over many humans obtained by pooling together trials from a large number of individual human subjects and treating this human pool as if it were acquired from a single behaving agent. Due to intersubject variability, we do not expect any given human or monkey subject to be perfectly consistent with this archetypal human (i.e., we do not expect it to have a human consistency of 1.0). Given current limitations of monkey psychophysics, we are not yet able to measure the behavior of very large number of monkey subjects at high resolution and consequently cannot directly estimate the human consistency of the corresponding "archetypal monkey" to the human pool. Rather, we indirectly estimated this value by first measuring human consistency as a function of number of individual monkey subjects pooled together $(n)$ and then extrapolating the human consistency estimate for pools of very large number of subjects (as $n$ approaches infinity). Extrapolations were done using least-squares fitting of an exponential function $\tilde{\rho}(n)=a+b \cdot e^{-c n}$ (see Fig. 4).

For each behavioral metric, we defined a "primate zone" as the range of human consistency values delimited by estimates $\tilde{\rho}_{M_{\infty}}$ and $\tilde{\rho}_{H_{\infty}}$ as lower and upper bounds respectively. $\tilde{\rho}_{M \infty}$ corresponds to the extrapolated estimate of human consistency of a large (i.e., infinitely many) pool of rhesus macaque monkeys; $\tilde{\rho}_{H \infty}$ is by definition equal to 1.0. Therefore, the primate zone defines a range of human consistency values that correspond to models that accurately capture the behavior of the human pool at least as well as an extrapolation of our monkey sample. In this work, we defined this range of human consistency values as the criterion for success for computational models of primate visual object recognition behavior.

To make a global statistical inference about whether models sampled from the $\mathrm{DCNN}_{\mathrm{IC}}$ subfamily meet or fall short of this criterion for success, we attempted to reject the hypothesis that, for a given behavioral metric, the human consistency of $\mathrm{DCNN}_{\mathrm{IC}}$ models is within the primate zone. To test this hypothesis, we estimated the empirical probability that the distribution of human consistency values, estimated over different model instances within this family, could produce human consistency values within the primate zone. Specifically, we estimated a $p$-value for each behavioral metric using the following procedure: We first estimated 
an empirical distribution of Fisher-transformed human consistency values for this model family (i.e., over all tested $\mathrm{DCNN}_{\mathrm{IC}}$ models and over all trial resampling of each $\mathrm{DCNN}_{\mathrm{IC}}$ model). From this empirical distribution, we fit a Gaussian kernel density function, optimizing the bandwidth parameter to minimize the mean squared error to the empirical distribution. This kernel density function was evaluated to compute a $p$-value by computing the cumulative probability of observing a human consistency value greater than or equal to the criterion of success (i.e., the Fisher transformed $\tilde{\rho}_{M \infty}$ value). This $p$-value indicates the probability that human consistency values sampled from the observed distribution would fall into the primate zone, with smaller $p$-values indicating stronger evidence against the hypothesis that the human consistency of DCNN models is within the primate zone.

\section{Results}

In the present work, we systematically compared the basic level core object recognition behavior of primates and state-of-the-art artificial neural network models using a series of behavioral metrics ranging from low to high resolution within a two-alternative forced choice match-to-sample paradigm. The behavior of each visual system, whether biological or artificial, was tested on the same 2400 images ( 24 objects, 100 images/object) in the same 276 interleaved binary object recognition tasks. Each system's behavior was characterized at multiple resolutions (see "Behavioral metrics and signatures" section in the Materials and Methods) and directly compared with the corresponding behavioral metric applied on the archetypal human (defined as the average behavior of a large pool of human subjects tested; see Materials and Methods). The overarching logic of this study was that, if two visual systems are equivalent, then they should produce statistically indistinguishable behavioral signatures with respect to these metrics. Specifically, our goal was to compare the behavioral signatures of visual system models with the corresponding behavioral signatures of primates.

\section{Object-level behavioral comparison}

We first examined the pattern of one-versus-all object-level behavior (B.O1 metric) computed across all images and possible distractors. Because we tested 24 objects here, the B.O1 signature was 24 dimensional. Figure $2 A$ shows the B.O1 signatures for the pooled human (pooling $n=1472$ human subjects), pooled monkey (pooling $n=5$ monkey subjects), and several DCNN IC $_{\text {mod- }}$ els as 24-dimensional vectors using a color scale. Each element of the vector corresponds to the system's discriminability of one object against all others that were tested (i.e., all other 23 objects). The color scales span each signature's full performance range and warm colors indicate lower discriminability. For example, red indicates that the tested visual system found the object corresponding to that element of the vector to be very challenging to discriminate from other objects (on average over all 23 discrimination tests and on average over all images). Figure $2 B$ directly compares the B.O1 signatures computed from the behavioral output of two visual system models, a pixel model (Fig. 2B, top) and a DCNN $\mathrm{IC}_{\mathrm{IC}}$ model (Inception-v3; Fig. $2 B$, bottom), against that of the human B.O1 signature. We observe a tighter correspondence to the human behavioral signature for the $\mathrm{DCNN}_{\mathrm{IC}}$ model visual system than for the baseline pixel model visual system. We quantified that similarity using a noise-adjusted correlation between each pair of B.O1 signatures (termed human consistency; Johnson et al., 2002). The noise adjustment means that a visual system that is identical to the human pool will have an expected human consistency score of 1.0 even if it has irreducible trial-by-trial stochasticity (see Materials and Methods). Figure $2 \mathrm{C}$ shows the B.O1 human consistency for each of the tested model visual systems. We additionally tested the behavior of a held-out pool of five human subjects (black dot) and a pool of five macaque monkey subjects (gray dot) and observed that both yielded B.O1 signatures that were highly human consistent (human consistency, $\tilde{\rho}=0.90,0.97$ for monkey pool and held-out human pool, respectively). We defined a range of human consistency values, termed the "primate zone" (shaded gray area), delimited by extrapolated human consistency estimates of large pools of macaques (see Materials and Methods; see Fig. 4). We found that the baseline pixel visual system model and the lowlevel V1 visual system model were not within this zone ( $\tilde{\rho}=0.40$, 0.67 for pixels and V1 models, respectively), whereas all tested $\mathrm{DCNN}_{\text {IC }}$ visual system models were either within or very close to this zone. Indeed, we could not reject the hypothesis that $\mathrm{DCNN}_{\mathrm{IC}}$ models are primate like ( $p=0.54$, exact test; see Materials and Methods).

Next, we compared the behavior of the visual systems at a slightly higher level of resolution. Specifically, instead of pooling over all discrimination tasks for each object, we computed the mean discriminability of each of the 276 pairwise discrimination tasks (still pooling over images within each of those tasks). This yielded a symmetric matrix that is referred to here as the B.O2 signature. Figure $2 \mathrm{D}$ shows the $\mathrm{B} . \mathrm{O} 2$ signatures of the pooled human, pooled monkey and several $\mathrm{DCNN}_{\mathrm{IC}}$ visual system models as $24 \times 24$ symmetric matrices. Each bin $(i, j)$ corresponds to the system's discriminability of objects $i$ and $j$, where warmer colors indicate lower performance; color scales are not shown but span each signature's full range. We observed strong qualitative similarities between the pairwise object confusion patterns of all of the high level visual systems (e.g., camel and dog are often confused with each other by all three systems). This similarity is quantified in Figure 2E, which shows the human consistency of all examined visual system models with respect to this metric. Similar to the B.O1 metric, we observed that both a pool of macaque monkeys and a held-out pool of humans are highly human consistent with respect to this metric ( $\tilde{\rho}=0.77,0.94$ for monkeys, humans respectively). Also similar to the B.O1 metric, we found that all DCNN $\mathrm{IC}_{\mathrm{IC}}$ visual system models are highly human consistent $(\tilde{\rho}>0.8)$, whereas the baseline pixel visual system model and the low-level V1 visual system model were not ( $\tilde{\rho}=0.41,0.57$ for pixels, V1 models, respectively). Indeed, all $\mathrm{DCNN}_{\text {IC }}$ visual system models are within the defined "primate zone" of human consistency and we could not falsify the hypothesis that DCNN $_{\text {IC }}$ models are primate like ( $p=0.99$, exact test).

Together, humans, monkeys, and current DCNN $_{\text {IC }}$ models all share similar patterns of object-level behavioral performances (B.O1 and B.O2 signatures) that are not shared with lower-level visual representations (pixels and V1). However, object-level performance patterns do not capture the fact that some images of an object are more challenging than other images of the same object because of interactions of the variation in the object's pose and position with the object's class. To overcome this limitation, we next examined the patterns of behavior at the resolution of individual images on a subsampled set of images where we specifically obtained a large number of behavioral trials to accurately estimate behavioral performance on each image. Note that, from the point of view of the subjects, the behavioral tasks are identical to those already described. We simply aimed to measure and compare their patterns of performance at much higher resolution.

\section{Image-level behavioral comparison}

To isolate purely image-level behavioral variance, that is, variance that is not predicted by the object and thus already captured 
A B.01 Signatures ( object difficulties)

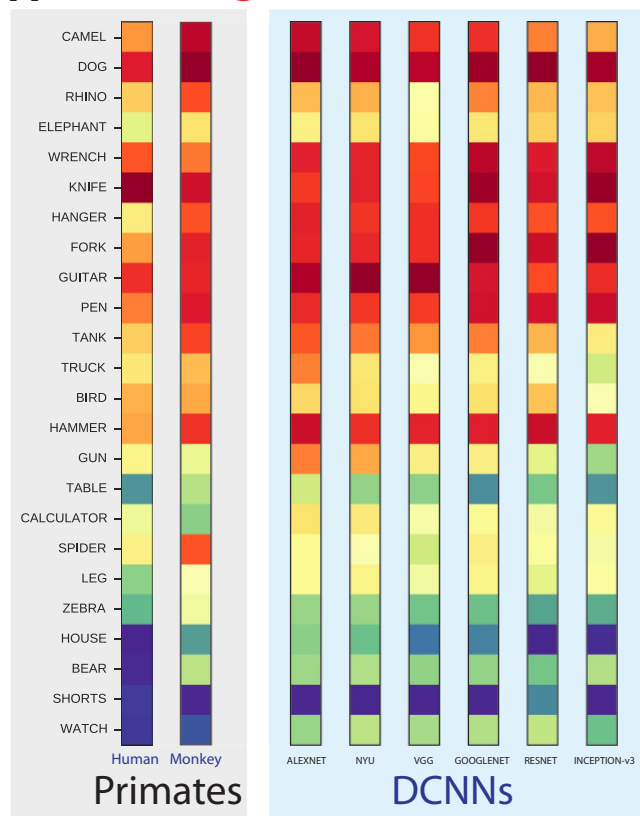

B
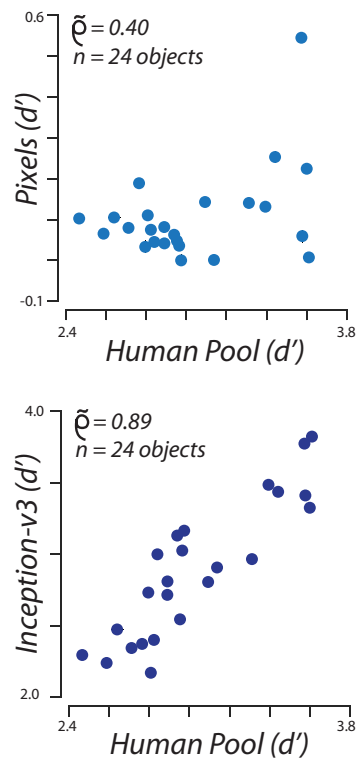

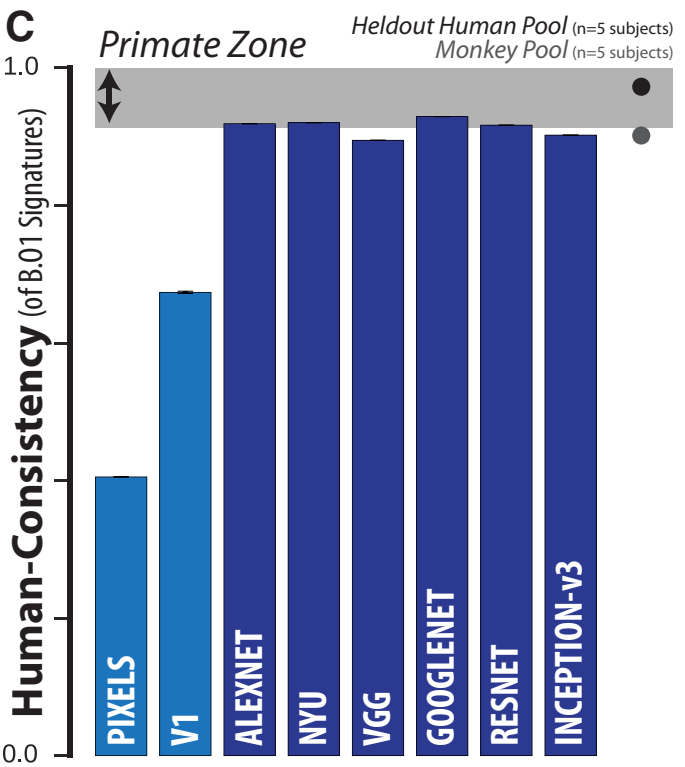

D B.02 Signatures ( object confusions)
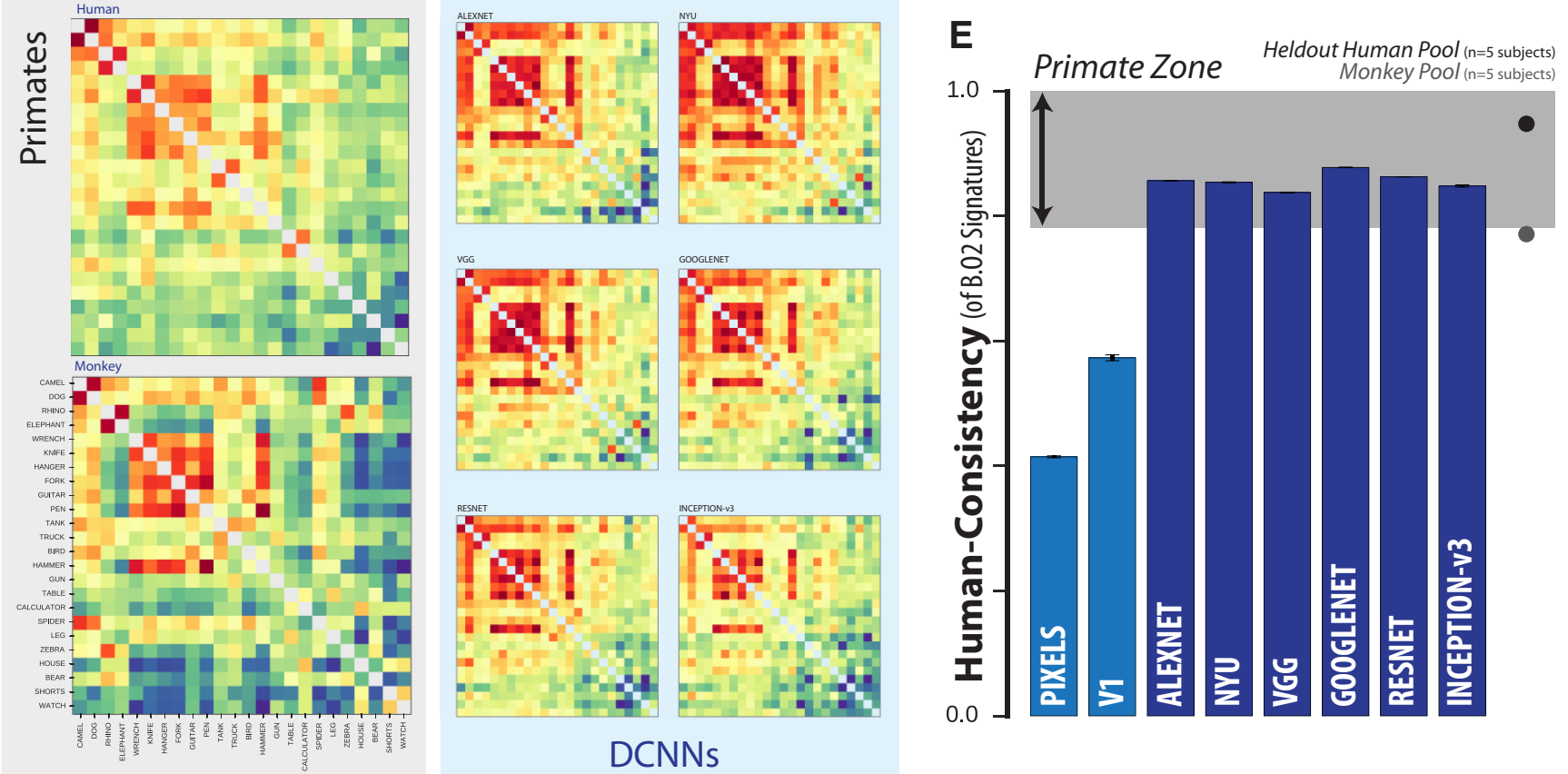

Figure 2. Object-level comparison to human behavior. A, One-versus-all object-level (B.01) signatures for the pooled human ( $n=1472$ human subjects), pooled monkey ( $n=5$ monkey subjects), and several DCNN ${ }_{1 C}$ models. Each B.01 signature is shown as a 24-dimensional vector using a color scale; each colored bin corresponds to the system's discriminability of one object against all others that were tested. The color scales span each signature's full performance range and warm colors indicate lower discriminability. $\boldsymbol{B}$, Direct comparison of the $B .01$ signatures of a pixel visual system model (top) and a DCNN ${ }_{1 C}$ visual system model (Inception-v3; bottom) against that of the human B.01 signature. C, Human consistency of B.01 signatures for each of the tested model visual systems. The black and gray dots correspond to a held-out pool of five human subjects and a pool of five macaque monkey subjects, respectively. The shaded area corresponds to the "primate zone," a range of consistencies delimited by the estimated human consistency of a pool of infinitely many monkeys (see Fig. 4A). D, One-versus-other object-level (B.02) signatures for pooled human, pooled monkey, and several DCNN ${ }_{1 C}$ models. Each B.02 signature is shown as a $24 \times 24$ symmetric matrices using a color scale, where each bin (i,j) corresponds to the system's discriminability of objects $i$ and $j$. As in $A$, color scales span each signature's full performance range and warm colors indicate lower discriminability. $E$, Human consistency of B.02 signatures for each of the tested model visual systems. Format is identical to that in $\mathbf{C}$.

by the B.O1 signature, we computed the normalized image-level signature. This normalization procedure is schematically illustrated in Figure $3 A$, which shows that the one-versus-all imagelevel signature (240-dimensional, 10 images/object) is used to obtain the normalized one-versus-all image-level signature (termed B.I1n, see "Behavioral metrics and signatures" section). Figure $3 B$ shows the B.I1n signatures for the pooled human, pooled monkey, and several $\mathrm{DCNN}_{\mathrm{IC}}$ models as 240 dimensional vectors. Each bin's color corresponds to the discriminability of a single image against all distractor options (after subtraction of 

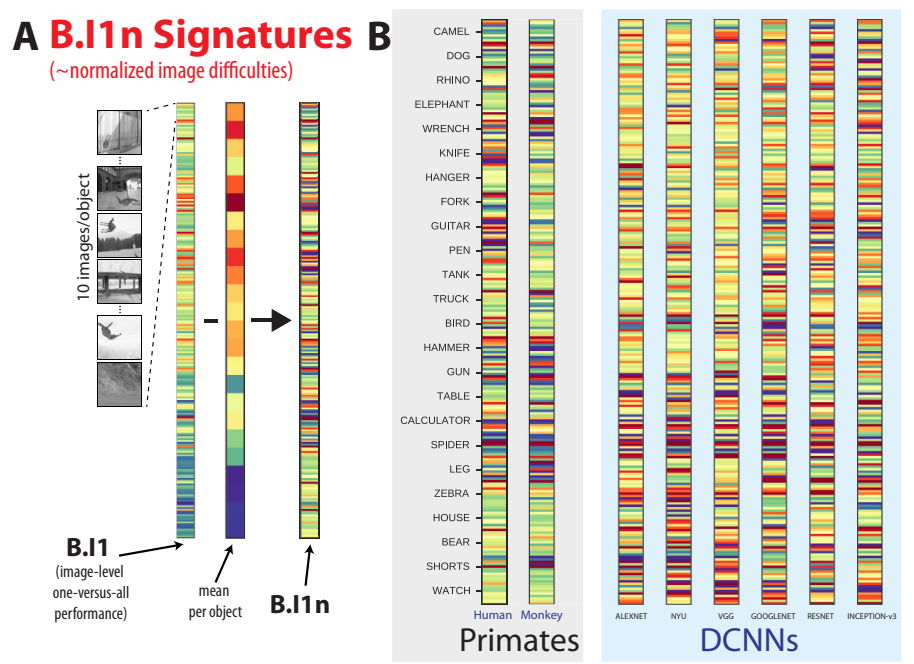

D

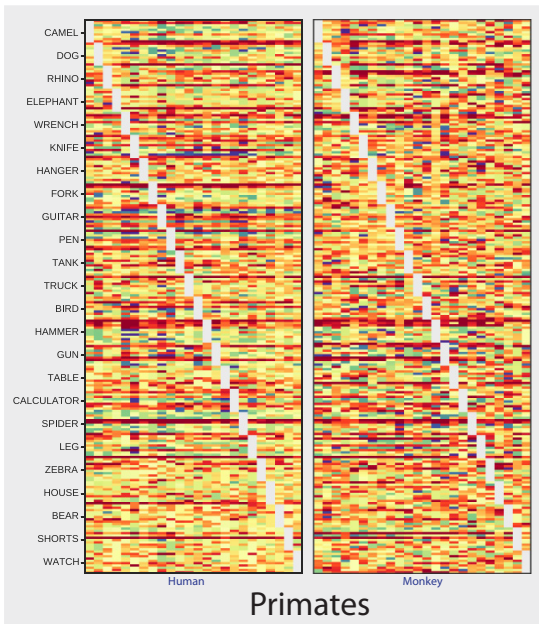

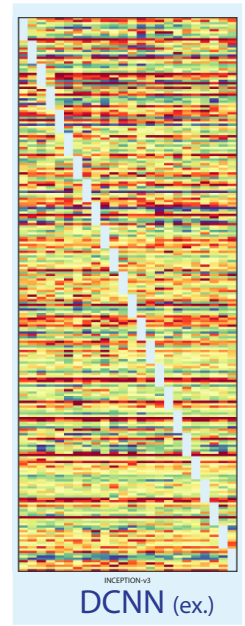

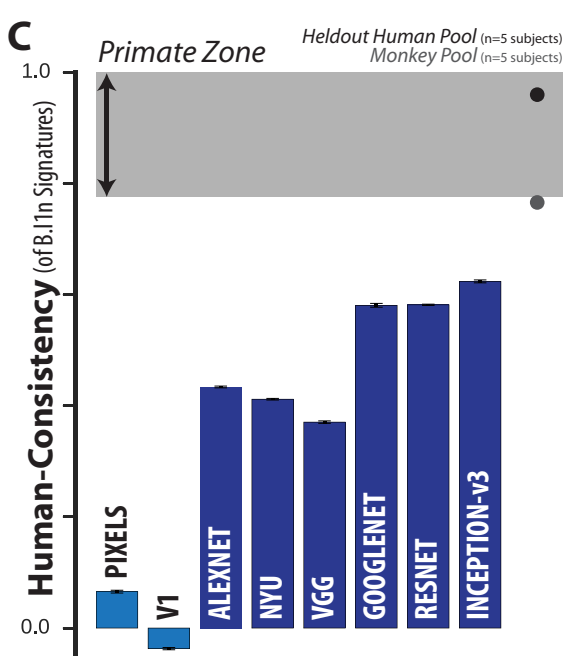

E

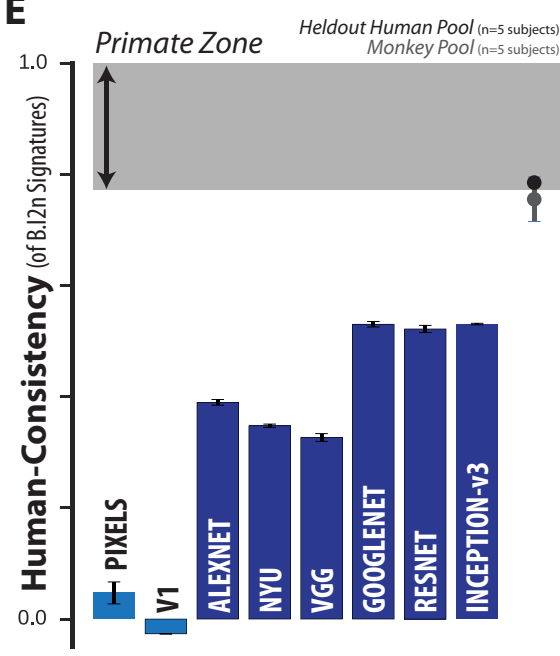

Figure 3. Image-level comparison to human behavior. A, Schematic for computing B.I1n. First, the one-versus-all image-level signature (B.I1) is shown as a 240-dimensional vector (24 objects, $10 \mathrm{images} / 0$ bject) using a color scale, where each colored bin corresponds to the system's discriminability of one image against all distractor objects. From this pattern, the normalized one-versus-all image-level signature (B.I1n) is estimated by subtracting the mean performance value over all images of the same object. This normalization procedure isolates behavioral variance that is specifically image driven but not simply predicted by the object. B, Normalized one-versus-all object-level (B.I1n) signatures for the pooled human, pooled monkey, and several DCNN ${ }_{1 C}$ models. Each B.I1n signature is shown as a 240-dimensional vector using a color scale formatted as in $A$. C, Human consistency of $B$.I1n signatures for each of the tested model visual systems. Format is identical to that in Figure 2C. D, Normalized one-versus-other image-level (B.I2n) signatures for pooled human, pooled monkey, and several DCNN ${ }_{1 C}$ models. Each B.I2n signature is shown as a $240 \times 24$ matrix using a color scale, where each bin $(i, j)$ corresponds to the system's discriminability of image i against distractor object $j$. Colors scales in $A, B$ and $D$ span each signature's full performance range and warm colors indicate lower discriminability. $\boldsymbol{E}$, Human consistency of B.I2n signatures for each of the tested model visual systems. Format is identical to that in Figure $2 C$.

object-level discriminability; see Fig. 3A), where warmer colors indicate lower values; color scales are not shown but span each signature's full range. Figure $3 C$ shows the human consistency with respect to the B.Inn signature for all tested models. Unlike with object-level behavioral metrics, we now observe a divergence between $\mathrm{DCNN}_{\mathrm{IC}}$ models and primates. Both the monkey pool and the held-out human pool remain highly human consistent ( $\tilde{\rho}=0.77,0.96$ for monkeys, humans respectively), but all $\mathrm{DCNN}_{\mathrm{IC}}$ models were significantly less human consistent (Inception-v3: $\tilde{\rho}=$ 0.62 ) and well outside of the defined "primate zone" of B.IIn human consistency. Indeed, the hypothesis that the human consistency of $\mathrm{DCNN}_{\mathrm{IC}}$ models is within the primate zone is strongly rejected ( $p=$ 6.16e-8, exact test; see Materials and Methods).

We can zoom in further by examining not only the overall performance for a given image but also the object confusions for each image, that is, the additional behavioral variation that is due, not only to the test image, but also to the interaction of that test image with the alternative (incorrect) object choice that is pro- vided after the test image (Fig. 1B). This is the highest level of behavioral accuracy resolution that our task design allows. In raw form, it corresponds to one-versus-other image-level confusion matrix, where the size of that matrix is the total number of images by the total number of objects (here, $240 \times 24)$. Each bin $(i, j)$ corresponds to the behavioral discriminability of a single image $i$ against distractor object $j$. Again, we isolate variance that is not predicted by object-level performance by subtracting the average performance on this binary task (mean over all images) to convert the raw matrix B.I2 above into the normalized matrix, referred to as B.I2n. Figure 3D shows the B.I2n signatures as $240 \times$ 24 matrices for the pooled human, pooled monkey, and top $\mathrm{DCNN}_{\mathrm{IC}}$ visual system models. Color scales are not shown but span each signature's full range; warmer colors correspond to images with lower performance in a given binary task relative to all images of that object in the same task. Figure $3 E$ shows the human consistency with respect to the B.I2n metric for all tested visual system models. Extending our observations using 
B.I1n, we observe a similar divergence between primates and $\mathrm{DCNN}_{\mathrm{IC}}$ visual system models on the matrix pattern of image-bydistractor difficulties (B.I2n). Specifically, both the monkey pool and held-out human pool remain highly human consistent $(\tilde{\rho}=0.75$, 0.77 for monkeys, humans respectively), whereas all tested $\mathrm{DCNN}_{\mathrm{IC}}$ models are significantly less human consistent (Inception-v3: $\tilde{\rho}=$ 0.53 ) falling well outside of the defined "primate zone" of B.I $2 \mathrm{n}$ human consistency values. Once again, the hypothesis that the human consistency of $\mathrm{DCNN}_{\mathrm{IC}}$ models is within the primate zone is strongly rejected ( $p=3.17 \mathrm{e}-18$, exact test; see Materials and Methods).

\section{Natural subject-to-subject variation}

For each behavioral metric (B.O1, BO2, B.I1n, BI2n), we defined a "primate zone" as the range of consistency values delimited by human consistency estimates $\tilde{\rho}_{M \infty}$ and $\tilde{\rho}_{H_{\infty}}$ as lower and upper bounds respectively. $\tilde{\rho}_{M \infty}$ corresponds to the extrapolated estimate of the human consistency of a large (i.e., infinitely many subjects) pool of rhesus macaque monkeys. Therefore, the fact that a particular tested visual system model falls outside of the primate zone can be interpreted as a failure of that visual system model to accurately predict the behavior of the archetypal human at least as well as the archetypal monkey.

However, from the above analyses, it is not yet clear whether a visual system model that fails to predict the archetypal human might nonetheless accurately correspond to one or more individual human subjects found within the natural variation of the human population. Given the difficulty of measuring individual subject behavior at the resolution of single images for large numbers of human and monkey subjects, we could not yet directly test this hypothesis. Instead, we examined it indirectly by asking whether an archetypal model - that is, a pool that includes an increasing number of model "subjects"—would approach the human pool. We simulated model intersubject variability by retraining a fixed DCNN architecture with a fixed training image set with random variation in the initial conditions and order of training images. This procedure results in models that can still perform the task but with slightly different learned weight values. We note that this procedure is only one possible choice of generating intersubject variability within each visual system model type, a choice that is an important open research direction that we do not address here. From this procedure, we constructed multiple trained model instances ("subjects") for a fixed DCNN architecture and asked whether an increasingly large pool of model "subjects" better captures the behavior of the human pool at least as well as a monkey pool. This post hoc analysis was conducted for the most human consistent DCNN architecture (Inception-v3).

Figure $4 A$ shows, for each of the four behavioral metrics, the measured human consistency of subject pools of varying size (number of subjects, $n$ ) of rhesus macaque monkeys (black) and ImageNet-trained Inception-v3 models (blue). The human consistency increases with growing number of subjects for both visual systems across all behavioral metrics. To estimate the expected human consistency for a pool of infinitely many monkey or model subjects, we fit an exponential function mapping $n$ to the mean human consistency values and obtained a parameter estimate for the asymptotic value (see Materials and Methods). We note that estimated asymptotic values are not significantly beyond the range of the measured data; the human consistency of a pool of five monkey subjects reaches within $97 \%$ of the human consistency of an estimated infinite pool of monkeys for all metrics, giving credence to the extrapolated human consistency values. This analysis suggests that, under this model of intersubject variability, a pool of Inception-v3 subjects accurately capture archetypal human behavior at the resolution of objects (B.O1, B.O2) by our primate zone criterion (Fig. $4 A$, first two panels). In contrast, even a large pool of Inception-v3 subjects still fails at its final asymptote to accurately capture human behavior at the image level (B.I1n, B.I2n) (Fig. 4A, last two panels).

\section{Modification of visual system models to try to rescue their human consistency}

Next, we wondered whether some relatively simple changes to the DCNN $_{\text {IC }}$ visual system models tested here could bring them into better correspondence with the primate visual system behavior (with respect to B.I1n and B.I2n metrics). Specifically, we considered and tested the following modifications to the most human consistent $\mathrm{DCNN}_{\mathrm{IC}}$ model visual system (Inception-v3): we did the following: (1) changed the input to the model to be more primate-like in its retinal sampling (Inception-v3 + retina-like), (2) changed the transformation (aka "decoder") from the internal model feature representation into the behavioral output by augmenting the number of decoder training images or changing the decoder type (Inception-v3 + SVM, Inception-v3 + classifier_train), and (3) modified all of the internal filter weights of the model (aka "fine tuning") by augmenting its ImageNet training with additional images drawn from the same distribution as our test images (Inception-v3 + synthetic-fine-tune), and (4) modified all of the internal filter weights of the model by training exclusively on images drawn from the same distribution as our test images (Inception-v3 + synthetic-train). All model modifications resulted in relatively high-performing models; Figure 5 (gray bars) shows the mean overall performance over object recognition tasks evaluated with a fixed decoder type (logistic classifier, 20 training images per class). However, we found that none of these modifications led to a significant improvement in its human consistency on the behavioral metrics (Fig. 4B). In particular, training exclusively on synthetic images led to a noted decrease in human consistency across all metrics. Figure 5 shows the relationship between model performances (B.O2, averaged over 276 tasks) and human consistency for both object-level and image-level metrics. We observe a strong correlation between performance and image-level human consistency across different model architectures trained on ImageNet (black points, $r=0.97$, $p<10^{-4}$ ), but no such correlation across our modified models within a fixed architecture (gray points, $r=0.35, p=0.13$ ). Therefore, the failure of current $\mathrm{DCNN}_{\mathrm{IC}}$ models to accurately capture the image-level signatures of primates cannot be rescued by simple modifications on a fixed architecture.

\section{Looking for clues: Image-level comparisons of models and primates}

Together, Figures 2, 3, 4, and 5 suggest that current DCNN $_{\text {IC }}$ visual system models fail to accurately capture the image-level behavioral signatures of humans and monkeys. To further examine this failure in the hopes of providing clues for model improvement, we examined the image-level residual signatures of all the visual system models relative to the pooled human. For each model, we computed its residual signature as the difference (positive or negative) of a linear least-squares regression of the model signature on the corresponding human signature. For this analysis, we focused on the B.In metric because it showed a clear divergence of $\mathrm{DCNN}_{\mathrm{IC}}$ models and primates and the behavioral residual can be interpreted based only on the test images (whereas B.I2n depends on the interaction between test images and distractor choice). 

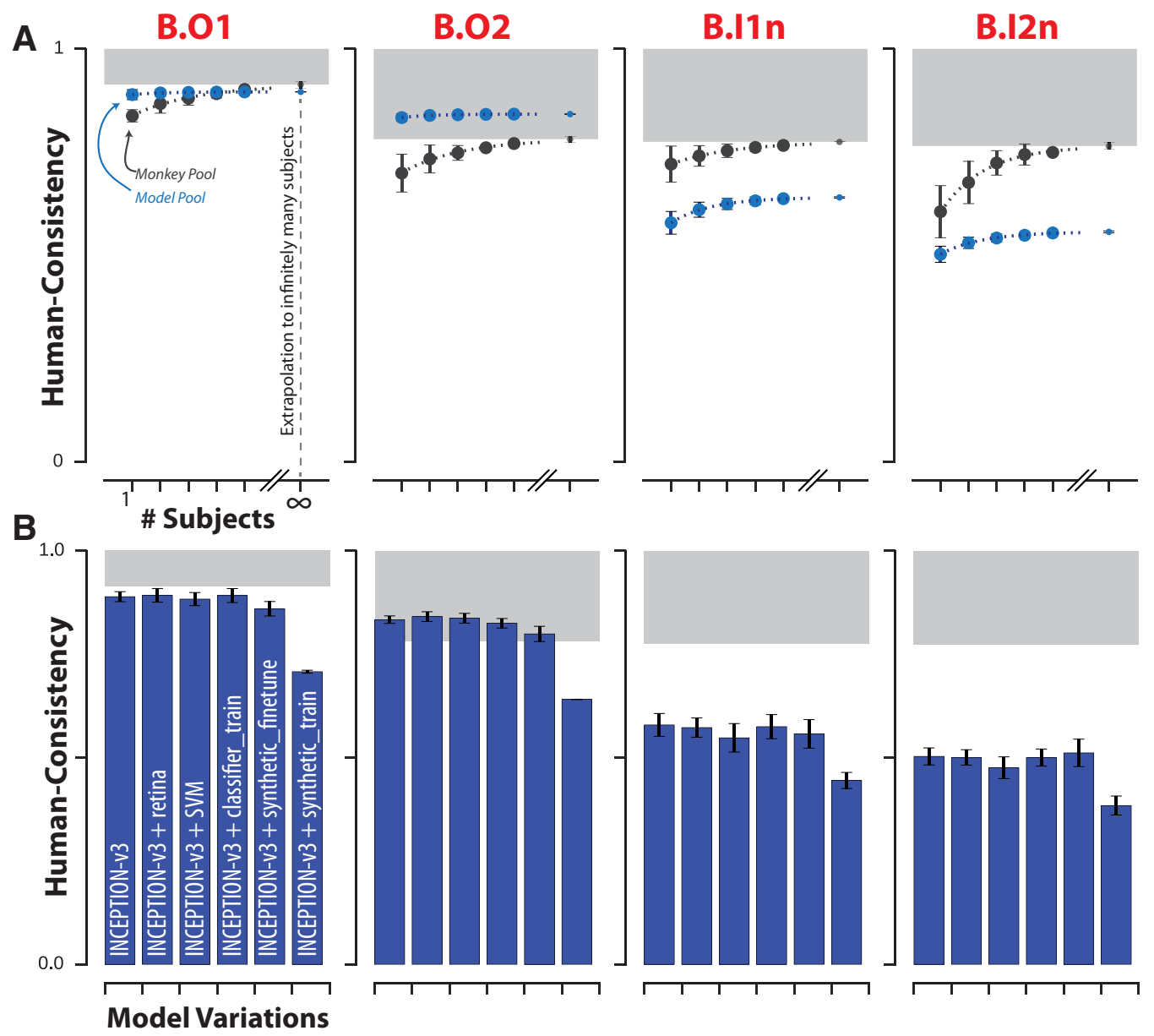

Figure 4. Effect of subject pool size and DCNN model modifications on consistency with human behavior. $A$, For each of the four behavioral metrics, the human consistency distributions of monkey (blue markers) and model (black markers) pools are shown as a function of the number of subjects in the pool (mean \pm SD, over subjects). Human consistency increases with growing number of subjects for all visual systems across all behavioral metrics. The dashed lines correspond to fitted exponential functions and the parameter estimate (mean \pm SE) of the asymptotic value, corresponding to the estimated human consistency of a pool of infinitely many subjects, is shown at the right most point on each abscissa. $\boldsymbol{B}$, Model modifications that aim to rescue the DCNN ${ }_{l C}$ models. We tested several simple modifications (see Materials and Methods) to the most human consistent DCNN ${ }_{1 C}$ visual system model (Inception-v3). Each panel shows the resulting human consistency per modified model (mean \pm SD. over different model instances, varying in random filter initializations) for each of the four behavioral metrics.

We first asked to what extent the residual signatures are shared between different visual system models. Figure $6 \mathrm{~A}$ shows the similarity between the residual signatures of all pairs of models; the color of bin $(i, j)$ indicates the proportion of explainable variance that is shared between the residual signatures of visual systems $i$ and $j$. For ease of interpretation, we ordered visual system models based on their architecture and optimization procedure and partitioned this matrix into four distinct regions. Each region compares the residuals of a "source" model group with fixed architecture and optimization procedure (five Inception-v3 models optimized for categorization on ImageNet, varying only in initial conditions and training image order) to a "target" model group. The target groups of models for each of the four regions are as follows: (1) the pooled monkey, (2) other $\mathrm{DCNN}_{\mathrm{IC}}$ models from the source group, (3) $\mathrm{DCNN}_{\mathrm{IC}}$ models that differ in architecture but share the optimization procedure of the source group models, and (4) $\mathrm{DCNN}_{\mathrm{IC}}$ models that differ slightly using an augmented optimization procedure but share the architecture of the source group models. Figure $6 B$ shows the mean $( \pm S D)$ variance shared in the residuals averaged within these four regions for all images (black dots), as well as for images that humans found to be particularly difficult (gray dots, selected based on held-out human data, see Materials and Methods). First, consistent with the results shown in Figure 3, we note that the residual signatures of this particular $\mathrm{DCNN}_{\mathrm{IC}}$ model are not well shared with the pooled monkey $\left(r^{2}=0.39\right.$ in region 1$)$ and this phenomenon is more pronounced for the images that humans found most difficult $\left(r^{2}=0.17\right.$ in region 1$)$. However, this relatively low correlation between model and primate residuals is not indicative of spurious model residuals because the model residual signatures were highly reliable between different instances of this fixed $\mathrm{DCNN}_{\mathrm{IC}}$ model across random training initializations (region 2: $r^{2}=0.79,0.77$ for all and most difficult images, respectively). Interestingly, residual signatures were still largely shared with other DCNN $\mathrm{IC}_{\mathrm{IC}}$ models with vastly different architectures (region $3: r^{2}=0.70,0.65$ for all and most difficult images, respectively). However, residual signatures were more strongly altered when the visual training diet of the same architecture was altered (region 4: $r^{2}=0.57,0.46$ for all and most difficult images respectively, cf. region 3). Together, these results indicate that the images where $\mathrm{DCNN}_{\mathrm{IC}}$ visual system models diverged from humans (and monkeys) were not spurious, but were rather highly reliable across different model architectures, demonstrating that current $\mathrm{DCNN}_{\mathrm{IC}}$ models systematically and similarly diverge from primates.

Figure $7 A$ shows example images sorted by B.I1n squared residuals, corresponding to images that models and primates 


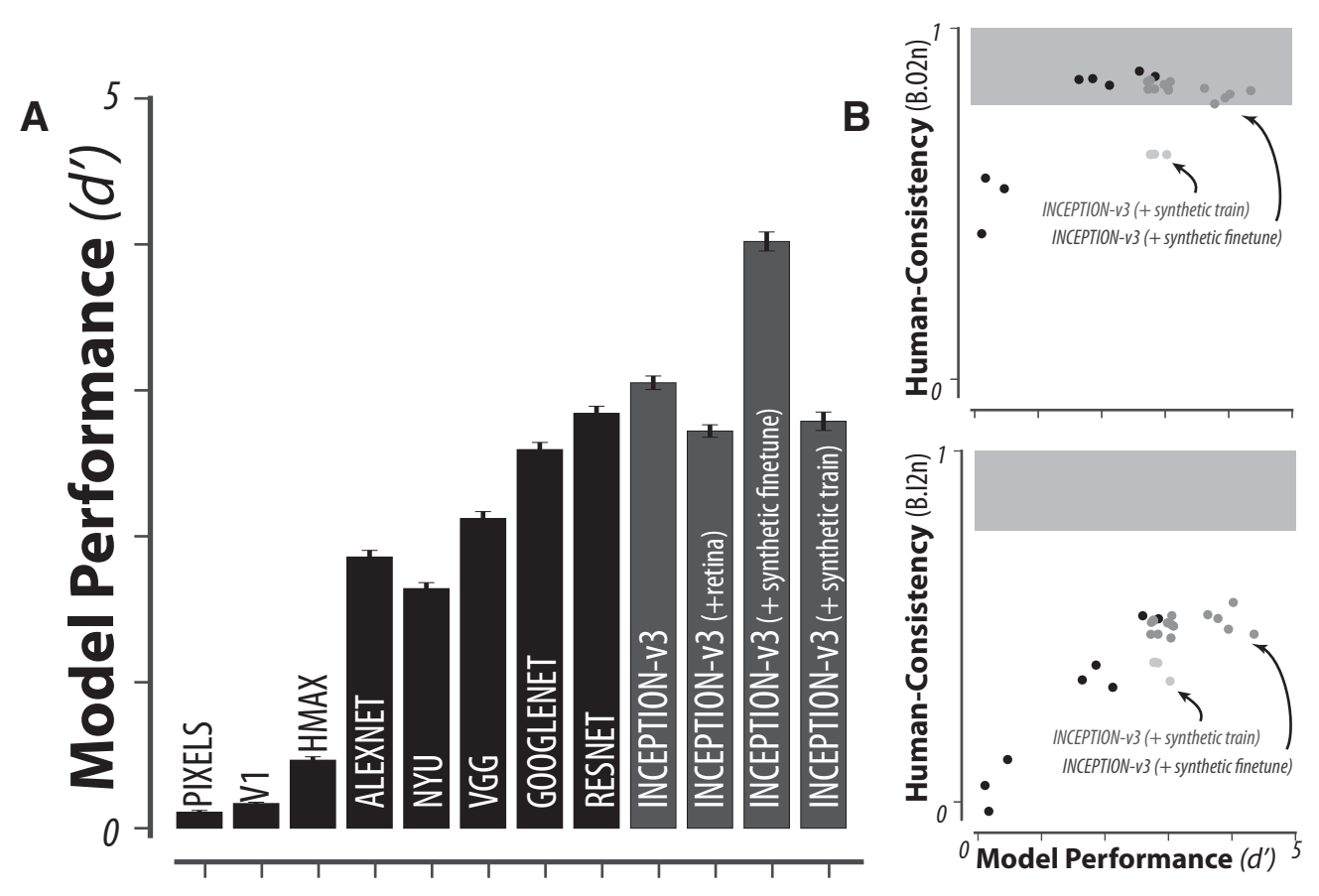

Figure 5. Model performance. A, Model performance on synthetic images (average B.02 across 276 tasks) for each of the tested models fixing the number of training images and classifier. Black bars correspond to different model architectures, with fixed optimization, whereas gray bars correspond to different modifications of a fixed model architecture (Inception-v3). $\boldsymbol{B}$, Correlation between model performance and human consistency with respect to object-level (B.02) and image-level (B.I2n) behavioral metrics. Each point corresponds to a single instance of a trained DCNN model.
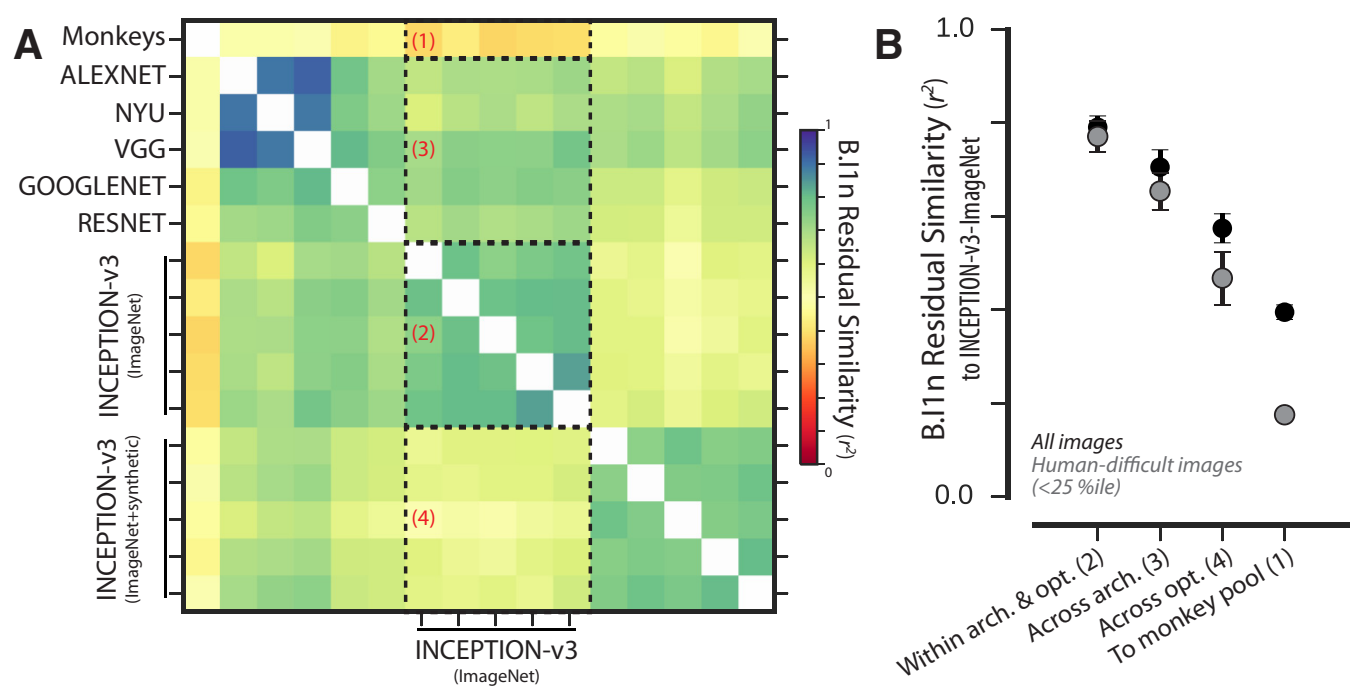

Figure 6. Analysis of unexplained human behavioral variance. $A$, Residual similarity between all pairs of human visual system models. The color of bin (i,j) indicates the proportion of explainable variance that is shared between the residual signatures of visual systems $i$ and $j$. For ease of interpretation, we ordered visual system models based on their architecture and optimization procedure and partitioned this matrix into four distinct regions. $\boldsymbol{B}$, Summary of residual similarity. For each of the four regions in Figure $5 A$, the similarity to the residuals of Inception-v 3 (region 2 in $\boldsymbol{A}$ ) is shown (mean \pm SD, within each region) for all images (black dots) and for images that humans found to be particularly difficult (gray dots, selected based on held-out human data).

largely agreed on (left) and diverged on (right) with respect to the B.Iln metric. We observed no qualitative differences between these images. To look for clues for model improvement, we asked what, if any, characteristics of images might account for this divergence of models and primates. We regressed the residual signatures of $\mathrm{DCNN}_{\mathrm{IC}}$ models on four different image attributes (corresponding to the size, eccentricity, pose, and contrast of the object in each image). We used multiple linear regressions to predict the model residual signatures from all of these image attributes and also considered each attribute individually using simple linear regressions. Figure $7 B$ shows example images (sampled from the full set of 2400 images) with increasing attribute value for each of these four image attributes. Although the $D_{C N N}$ IC models were not directly optimized to display primatelike performance dependence on such attributes, we observed that the Inception-v3 visual system model nonetheless exhibited qualitatively similar performance dependencies as primates (Fig. $7 C$ ). For example, humans (black), monkeys (gray), and the Inception-v3 model (blue) all performed better, on average, for images in which the object is in the center of gaze (low eccentricity) and large in size. Furthermore, all three systems performed better, on average, for images when the pose of the object was closer to the canonical pose (Fig. $7 \mathrm{C}$ ); this sensitivity to object pose manifested itself as a nonlinear dependence due to the fact 
A
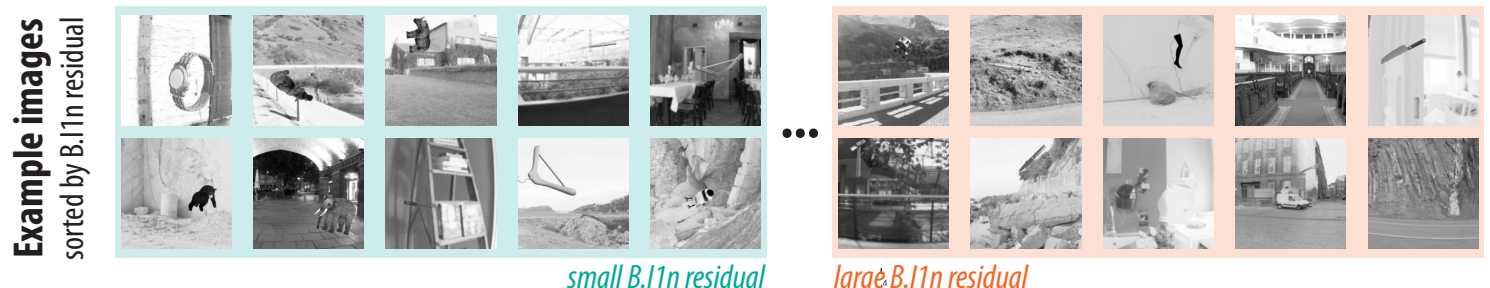

large. B.IIn residual
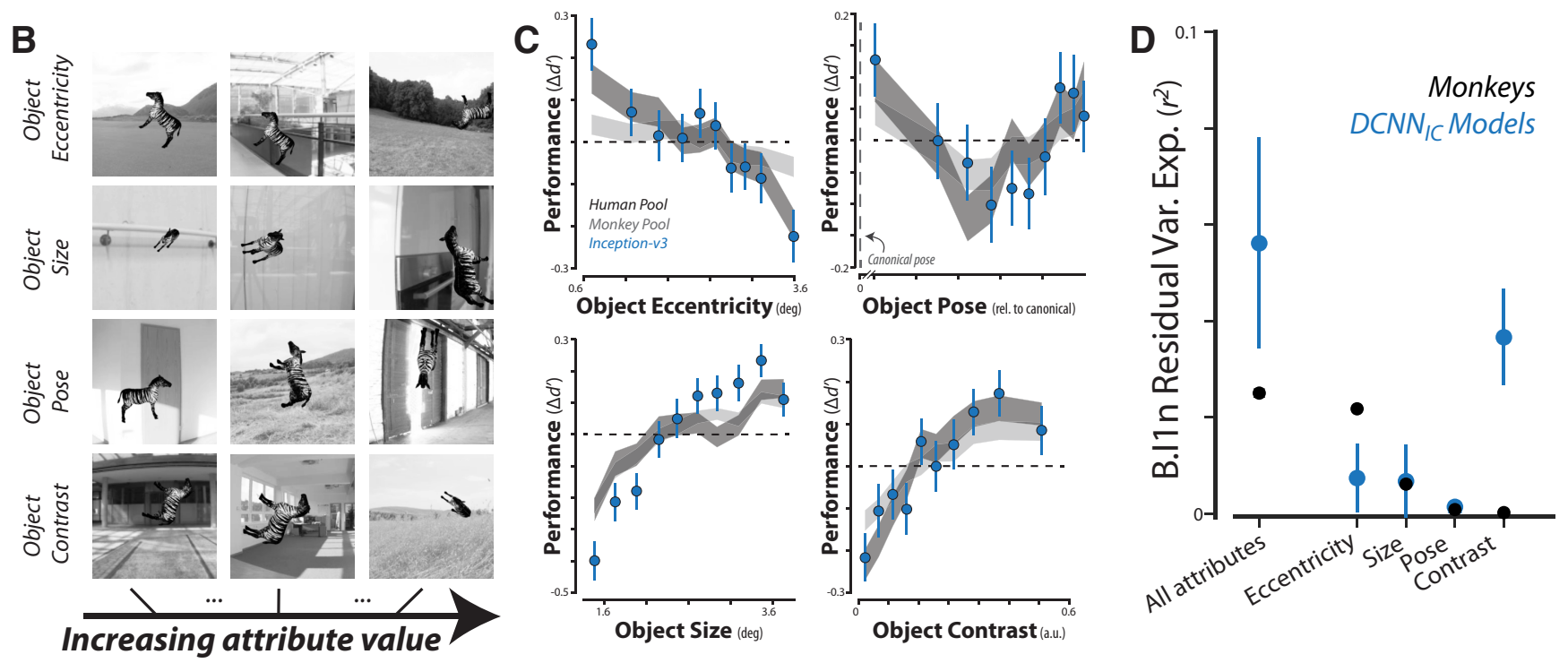

Figure 7. Dependence of primate and DCNN model behavior on image attributes. A, Example images showing that models and primates agree on (left) and diverge on (right) with respect to B.I1n residuals. $B$, Example images with increasing attribute value for each of the four predefined image attributes (see Materials and Methods). $C$, Dependence of performance (B.I1n) as a function of four image attributes for humans, monkeys, and a DCNN ${ }_{\mathrm{IC}}$ model (Inception-v3). $\boldsymbol{D}$, Proportion of explainable variance of the residual signatures of monkeys (black) and DCNN $\mathrm{I}_{\mathrm{IC}}$ models (blue) that is accounted for by each of the predefined image attributes. Error bars correspond to SD over trial resampling for monkeys and over different models for DCNN ${ }_{I C}$ models. .

that all tested objects exhibited symmetry in at least one axis. The similarity of the patterns in Figure $7 C$ between primates and the $\mathrm{DCNN}_{\mathrm{IC}}$ visual system models is not perfect but is striking, particularly in light of the fact that these models were not optimized to produce these patterns. However, this similarity is analogous to the similarity in the B.O1 and B.O2 metrics in that it only holds on average over many images. Looking more closely at the imageby-image comparison, we again found that the $\mathrm{DCNN}_{\mathrm{IC}}$ models failed to capture a large portion of the image-by-image variation (Fig. 3). In particular, Figure $7 D$ shows the proportion of variance explained by specific image attributes for the residual signatures of monkeys (black) and $\mathrm{DCNN}_{\mathrm{IC}}$ models (blue). We found that, together, all four of these image attributes explained only $\sim 10 \%$ of the variance in $\mathrm{DCNN}_{\mathrm{IC}}$ residual signatures and each individual attribute could explain at most a small amount of residual variance $(<5 \%$ of the explainable variance). In sum, these analyses show that some behavioral effects that might provide intuitive clues to modify the $\mathrm{DCNN}_{\mathrm{IC}}$ models are already in place in those models (e.g., a dependence on eccentricity). However, the quantitative image-by-image analyses of the remaining unexplained variance (Fig. $7 D$ ) argue that the $\mathrm{DCNN}_{\text {IC }}$ visual system models' failure to capture primate image-level signatures cannot be further accounted for by these simple image attributes and likely stem from other factors.

\section{Discussion}

The current work was motivated by the broad scientific goal of discovering models that quantitatively explain the neuronal mechanisms underlying primate invariant object recognition behavior. To this end, previous work had shown that specific ANNs drawn from a large family of DCNNs and optimized to achieve high levels of object categorization performance on large-scale image sets capture a large fraction of the variance in primate visual recognition behaviors (Ghodrati et al., 2014; Rajalingham et al., 2015; Jozwik et al., 2016; Kheradpisheh et al., 2016; Kubilius et al., 2016; Peterson et al., 2016; Battleday et al., 2017; Wallis et al., 2017) and the internal hidden neurons of those same models also predict a large fraction of the image-driven response variance of brain activity at multiple stages of the primate ventral visual stream (Yamins et al., 2013, 2014; Cadieu et al., 2014; KhalighRazavi and Kriegeskorte, 2014; Güçlü and van Gerven, 2015; Cichy et al., 2016, 2017; Hong et al., 2016; Seibert et al., 2016; Cadena et al., 2017; Eickenberg et al., 2017; Khaligh-Razavi et al., 2017; Seeliger et al., 2017; Wen et al., 2017). For clarity, we here referred to this subfamily of models as $\mathrm{DCNN}_{\mathrm{IC}}$ (to denote ImageNet categorization training) to distinguish them from all possible models in the DCNN family and, more broadly, from the superfamily of all ANNs. In this work, we directly compared leading $\mathrm{DCNN}_{\mathrm{IC}}$ models to primates (humans and monkeys) with respect to their behavioral signatures at both object-level and image-level resolution in the domain of core object recognition. To do so, we measured and characterized primate behavior at larger scale and higher resolution than previously possible. We first replicate prior work (Rajalingham et al., 2015) showing that, at the object level, $\mathrm{DCNN}_{\mathrm{IC}}$ models produce statistically indistinguishable behavior from primates and we extend that work by showing that these models also match the average primate sensitivities to object contrast, eccentricity, size, and pose, a noteworthy similarity in light of the fact that these models were not optimized 
to produce these performance patterns. This similarity, which we speculate may largely reflect the training history of these models (i.e., optimization on photographer-framed images, which may be biased with respect to these attributes), is a good direction for future work. However, our primary novel result is that, examining behavior at the higher resolution of individual images, all leading $\mathrm{DCNN}_{\mathrm{IC}}$ models failed to replicate the image-level behavioral signatures of primates. An important related claim is that rhesus monkeys are more consistent with the archetypal human than any of the tested $\mathrm{DCNN}_{\mathrm{IC}}$ models (at the image level).

We compared human, monkey, and model behavior on a set of naturalistic synthetic images sampled with systematic variation in viewpoint parameters and uncorrelated background. Although these images may seem non-natural, synthetic images of this types are, unlike some photographic image sets, very powerful at separating humans from low-level computer vision systems (Pinto et al., 2008). Furthermore, given our definition of success (a candidate visual system model that accurately captures primate behavior for all images), the inference that models differ from primates can be supported by testing models on any image set, including the synthetic set that we used. Importantly, this inference is with respect to a trained model's behavior at "run time," agnostic to its learning procedure. Although we do not make any claims about learning, we note that neither humans nor monkeys were separately optimized to perform on our synthetic images (beyond the minimal training described in the Materials and Methods section). Interestingly, we observed that syntheticimage-optimized models (new ANN models constructed by finetuning or training an existing network architecture exclusively on large sets of synthetic image) were no more similar to primates than ANN models optimized only on ImageNet, suggesting that the tested ANN architectures have one or more fundamental flaws that cannot be readily overcome by manipulating the training environment. Together, these results support the general inference, agnostic to natural choices of image set, that $\mathrm{DCNN}_{\mathrm{IC}}$ models diverge from primates in their core object recognition behavior.

This inference is consistent with previous work showing that DCNN $_{\text {IC }}$ models can diverge from human behavior on specifically chosen adversarial images (Szegedy et al., 2013). A strength of our work is that we did not optimize images to induce failure, but instead randomly sampled a broadly defined image generative parameter space highlighting a general rather than adversarial-induced divergence of $\mathrm{DCNN}_{\mathrm{IC}}$ from primate core object recognition behavior. Furthermore, we showed that this failure could not be rescued by a number of simple modifications to the model class, providing qualitative insight into what does and does not explain the gap between primates and DCNN models. Together, these results suggest that new ANN models are needed to more precisely capture the neural mechanisms underlying primate object vision.

With regard to new ANN models, we can attempt to make prospective inferences about future possible $\mathrm{DCNN}_{\mathrm{IC}}$ models from the data presented here. Based on the observed distribution of image-level human consistency values for the $\mathrm{DCNN}_{\mathrm{IC}}$ models tested here, we infer that as yet untested model instances sampled identically (i.e., from the $\mathrm{DCNN}_{\mathrm{IC}}$ model subfamily) are very likely to have similarly inadequate image-level human consistency. Although we cannot rule out the possibility that at least one model instance within the $\mathrm{DCNN}_{\mathrm{IC}}$ subfamily would fully match the image-level behavioral signatures, the probability of sampling such a model is vanishingly small $\left(p<10^{-17}\right.$ for B.I2n human consistency, estimated using exact test using Gaussian kernel density estimation; see Materials and Methods and Results). An important caveat of this inference is that we may have a biased estimate of the human consistency distribution of this model subfamily because we did not exhaustively sample the subfamily. In particular, if the model sampling process is nonstationary over time (e.g., increases in computational power over time allows larger models to be successfully trained), then the human consistency of new (i.e., yet to be sampled) models may lie outside the currently estimated distribution. Therefore, it is possible that the evolution of "next-generation" models within the DCNN $\mathrm{IC}_{\text {IC }}$ subfamily could meet our criteria for successful matching primate-like behavior.

Alternatively, it is possible-and we think likely-that future $\mathrm{DCNN}_{\text {IC }}$ models will also fail to capture primate-like image-level behavior, suggesting that either the architectural limitations (e.g., convolutional, feedforward) and/or the optimization procedure (including the diet of visual images) that define this model subfamily are fundamentally limiting. Therefore, ANN model subfamilies using different architectures (e.g., recurrent neural networks) and/or optimized for different behavioral goals (e.g., loss functions other than object classification performance, and/or images other than category-labeled ImageNet images) may be necessary to accurately capture primate behavior. To this end, we propose that testing even individual changes to the DCNN $_{\text {IC }}$ models, each creating a new ANN model subfamily, may be the best way forward because $\mathrm{DCNN}_{\mathrm{IC}}$ models currently offer the best explanations (in a predictive sense) of both the behavioral and neural phenomena of core object recognition.

To reach that goal of finding a new ANN model subfamily that is a better mechanistic model of the primate ventral visual stream, we propose that even larger-scale, high-resolution behavioral measurements such as expanded versions of the patterns of image-level performance presented here could serve as useful top-down optimization guides. Not only do these highresolution behavioral signatures have the statistical power to reject the currently leading ANN models, but they can also be efficiently collected at very large scale, in contrast to other guide data (e.g., large-scale neuronal measurements). Indeed, current technological tools for high-throughput psychophysics in humans and monkeys (e.g., MTurk for humans, MonkeyTurk for rhesus monkeys) enable time- and cost-efficient collection of large-scale behavioral datasets such as the $\sim 1$ million behavioral trials obtained for the current work. These systems trade off an increase in efficiency with a decrease in experimental control. For example, we did not impose experimental constraints on subjects' acuity and we can only infer likely head and gaze position. Previous work has shown that patterns of behavioral performance on object recognition tasks from in-laboratory and online subjects were equally reliable and virtually identical (Majaj et al., 2015), but it is not yet clear to what extent this holds at the resolution of individual images because one might expect that variance in performance across images is more sensitive to precise head and gaze location. For this reason, we here refrain from making strong inferences from small behavioral differences such as the small difference between humans and monkeys. Nevertheless, we argue that this sacrifice in exact experimental control while retaining sufficient power for model comparison is a good tradeoff for efficiently collecting large behavioral datasets toward the goal of constraining future models of the primate ventral visual stream.

\section{References}

Battleday RM, Peterson JC, Griffiths TL (2017) Modeling human categorization of natural images using deep feature representations. arXiv preprint arXiv:171104855. Advance online publication. Retrieved Nov 13, 2017. Available at https://arxiv.org/abs/1711.04855. 
Cadena SA, Denfield GH, Walker EY, Gatys LA, Tolias AS, Bethge M, Ecker AS (2017) Deep convolutional models improve predictions of macaque V1 responses to natural images. bioRxiv:201764. CrossRef

Cadieu CF, Hong H, Yamins DL, Pinto N, Ardila D, Solomon EA, Majaj NJ, DiCarlo JJ (2014) Deep neural networks rival the representation of primate IT cortex for core visual object recognition. PLoS Comput Biol 10:e1003963. CrossRef Medline

Cichy RM, Khosla A, Pantazis D, Torralba A, Oliva A (2016) Comparison of deep neural networks to spatio-temporal cortical dynamics of human visual object recognition reveals hierarchical correspondence. Sci Rep 6:27755. CrossRef Medline

Cichy RM, Khosla A, Pantazis D, Oliva A (2017) Dynamics of scene representations in the human brain revealed by magnetoencephalography and deep neural networks. Neuroimage 153:346-358. CrossRef Medline

DiCarlo JJ, Cox DD (2007) Untangling invariant object recognition. Trends Cogn Sci 11:333-341. CrossRef

DiCarlo JJ, Johnson KO (1999) Velocity invariance of receptive field structure in somatosensory cortical area $3 \mathrm{~b}$ of the alert monkey. J Neurosci 19:401-419. CrossRef Medline

DiCarlo JJ, Zoccolan D, Rust NC (2012) How does the brain solve visual object recognition? Neuron 73:415-434. CrossRef Medline

Dodge S, Karam L (2017) A study and comparison of human and deep learning recognition performance under visual distortions. arXiv preprint arXiv:170502498. Advance online publication. Retrieved May 6, 2017. Available at https://arxiv.org/abs/1705.02498

Eickenberg M, Gramfort A, Varoquaux G, Thirion B (2017) Seeing it all: convolutional network layers map the function of the human visual system. Neuroimage 152:184-194. CrossRef Medline

Geirhos R, Janssen DH, Schütt HH, Rauber J, Bethge M, Wichmann FA (2017) Comparing deep neural networks against humans: object recognition when the signal gets weaker. arXiv preprint arXiv:170606969. Advance online publication. Retrieved June 21, 2017. Available at https://arxiv.org/abs/1706.06969.

Ghodrati M, Farzmahdi A, Rajaei K, Ebrahimpour R, Khaligh-Razavi S-M (2014) Feedforward object-vision models only tolerate small image variations compared to human. Front Comput Neurosci 8:74. Medline

Goodfellow IJ, Shlens J, Szegedy C (2014) Explaining and harnessing adversarial examples. arXiv preprint arXiv:14126572. Advance online publication. Retrieved Dec 20, 2014. Available at https://arxiv.org/abs/1412.6572.

Güçlü U, van Gerven MA (2015) Deep neural networks reveal a gradient in the complexity of neural representations across the ventral stream. J Neurosci 35:10005-10014. CrossRef Medline

He K, Zhang X, Ren S, Sun J (2016) Deep residual learning for image recognition. In: Proceedings of the IEEE conference on computer vision and pattern recognition, pp 770-778.

Hong H, Yamins DL, Majaj NJ, DiCarlo JJ (2016) Explicit information for category-orthogonal object properties increases along the ventral stream. Nat Neurosci 19:613-622. CrossRef Medline

Hosseini H, Xiao B, Jaiswal M, Poovendran R (2017) On the limitation of convolutional neural networks in recognizing negative images. Human Performance 4:6.

Huynh DQ (2009) Metrics for 3D rotations: comparison and analysis. Journal of Mathematical Imaging and Vision 35:155-164. CrossRef

Johnson KO, Hsiao SS, Yoshioka T (2002) Neural coding and the basic law of psychophysics. Neuroscientist 8:111-121. CrossRef Medline

Jozwik KM, Kriegeskorte N, Mur M (2016) Visual features as stepping stones toward semantics: explaining object similarity in IT and perception with non-negative least squares. Neuropsychologia 83:201-226. CrossRef Medline

Khaligh-Razavi S-M, Kriegeskorte N (2014) Deep supervised, but not unsupervised, models may explain IT cortical representation. PLoS Comput Biol 10:e1003915. CrossRef Medline

Khaligh-Razavi S-M, Henriksson L, Kay K, Kriegeskorte N (2017) Fixed versus mixed RSA: explaining visual representations by fixed and mixed feature sets from shallow and deep computational models. Journal of Mathematical Psychology 76:184-197. CrossRef Medline

Kheradpisheh SR, Ghodrati M, Ganjtabesh M, Masquelier T (2016) Deep networks can resemble human feed-forward vision in invariant object recognition. Sci Rep 6:32672. CrossRef Medline

Kriegeskorte N (2015) Deep neural networks: a new framework for modeling biological vision and brain information processing. Annu Rev Vis Sci 1:417-446. CrossRef Medline
Krizhevsky A, Sutskever I, Hinton GE (2012) Imagenet classification with deep convolutional neural networks. In: Advances in neural information processing systems, pp 1097-1105.

Kubilius J, Bracci S, de Beeck HPO (2016) Deep neural networks as a computational model for human shape sensitivity. PLoS Comput Biol 12: e1004896. CrossRef Medline

LeCun Y, Bengio Y, Hinton G (2015) Deep learning. Nature 521:436-444. CrossRef Medline

Majaj NJ, Hong H, Solomon EA, DiCarlo JJ (2015) Simple learned weighted sums of inferior temporal neuronal firing rates accurately predict human core object recognition performance. J Neurosci 35:13402-13418. CrossRef Medline

Nguyen A, Yosinski J, Clune J (2015) Deep neural networks are easily fooled: High confidence predictions for unrecognizable images. In: Proceedings of the IEEE Conference on Computer Vision and Pattern Recognition, pp 427-436.

Peterson JC, Abbott JT, Griffiths TL (2016) Adapting deep network features to capture psychological representations. arXiv preprint arXiv:160802164. Advance online publication. Retrieved August 6, 2016. Available at https://arxiv.org/abs/1608.02164.

Pinto N, Cox DD, DiCarlo JJ (2008) Why is real-world visual object recognition hard? PLoS Comput Biol 4:e27. CrossRef Medline

Rajalingham R, Schmidt K, DiCarlo JJ (2015) Comparison of object recognition behavior in human and monkey. J Neurosci 35:12127-12136. CrossRef Medline

RichardWebster B, Anthony SE, Scheirer WJ (2016) PsyPhy: a psychophysics driven evaluation framework for visual recognition. arXiv preprint arXiv:161106448. Advance online publication. Retrieved Nov 19, 2016. Available at https://arxiv.org/abs/1611.06448.

Rolls ET (2000) Functions of the primate temporal lobe cortical visual areas in invariant visual object and face recognition. Neuron 27:205-218. CrossRef Medline

Rosch E, Mervis CB, Gray WD, Johnson DM, Boyes-Braem P (1976) Basic objects in natural categories. Cogn Psychol 8:382-439. CrossRef

Seeliger K, Fritsche M, Güçlü U, Schoenmakers S, Schoffelen J-M, Bosch S, van Gerven M (2017) Convolutional neural network-based encoding and decoding of visual object recognition in space and time. Neuroimage pii: S1053-8119(17)30586-4. CrossRef Medline

Seibert D, Yamins DL, Ardila D, Hong H, DiCarlo JJ, Gardner JL (2016) A performance-optimized model of neural responses across the ventral visual stream. bioRxiv:036475. CrossRef

Simonyan K, Zisserman A (2014) Very deep convolutional networks for large-scale image recognition. arXiv preprint arXiv:14091556. Advance online publication. Retrieved September 4, 2014. Available at https://arxiv.org/abs/1409.1556.

Szegedy C, Zaremba W, Sutskever I, Bruna J, Erhan D, Goodfellow I, Fergus R (2013) Intriguing properties of neural networks. arXiv preprint arXiv: 13126199. Advance online publication. Retrieved December 21, 2013. Available at https://arxiv.org/abs/1312.6199.

Tanaka K (1996) Inferotemporal cortex and object vision. Annu Rev Neurosci 19:109-139. CrossRef Medline

Ullman S, Humphreys GW (1996) High-level vision: object recognition and visual cognition. Cambridge, MA: MIT.

Wallis TS, Funke CM, Ecker AS, Gatys LA, Wichmann FA, Bethge M (2017) A parametric texture model based on deep convolutional features closely matches texture appearance for humans. J Vis 17:5. CrossRef Medline

Wen H, Shi J, Zhang Y, Lu KH, Cao J, Liu Z (2017) Neural encoding and decoding with deep learning for dynamic natural vision. Cereb Cortex 20:1-25. CrossRef Medline

Yamins DL, DiCarlo JJ (2016) Using goal-driven deep learning models to understand sensory cortex. Nat Neurosci 19:356-365. CrossRef Medline

Yamins DL, Hong H, Cadieu C, DiCarlo JJ (2013) Hierarchical modular optimization of convolutional networks achieves representations similar to macaque IT and human ventral stream. In: Advances in neural information processing systems, pp 3093-3101.

Yamins DL, Hong H, Cadieu CF, Solomon EA, Seibert D, DiCarlo JJ (2014) Performance-optimized hierarchical models predict neural responses in higher visual cortex. Proc Natl Acad Sci U S A 111:8619-8624. CrossRef Medline

Zeiler MD, Fergus R (2014) Visualizing and understanding convolutional networks. In: Computer Vision ECCV 2014, pp 818-833. New York, NY: Springer. 